\title{
Article \\ On Time-Periodic Bifurcation of a Sphere Moving under Gravity in a Navier-Stokes Liquid
}

\author{
Giovanni P. Galdi
}

Citation: Galdi, G.P. On

Time-Periodic Bifurcation of a Sphere Moving under Gravity in a Navier-Stokes Liquid. Mathematics 2021, 9, 715. https://doi.org/ $10.3390 /$ math 9070715

Academic Editor: Marco Pedroni

Received: 25 February 2021

Accepted: 11 March 2021

Published: 25 March 2021

Publisher's Note: MDPI stays neutral with regard to jurisdictional claims in published maps and institutional affiliations.

Copyright: (C) 2021 by the author. Licensee MDPI, Basel, Switzerland. This article is an open access article distributed under the terms and conditions of the Creative Commons Attribution (CC BY) license (https:// creativecommons.org/licenses/by/ $4.0 /)$.
Department of Mechanical Engineering and Materials Science, University of Pittsburgh, Pittsburgh, PA 15260, USA; galdi@pitt.edu

\begin{abstract}
We provide sufficient conditions for the occurrence of time-periodic Hopf bifurcation for the coupled system constituted by a rigid sphere, $\mathcal{S}$, freely moving under gravity in a Navier-Stokes liquid. Since the region of flow is unbounded (namely, the whole space outside $\mathcal{S}$ ), the main difficulty consists in finding the appropriate functional setting where general theory may apply. In this regard, we are able to show that the problem can be formulated as a suitable system of coupled operator equations in Banach spaces, where the relevant operators are Fredholm of index 0. In such a way, we can use the theory recently introduced by the author and give sufficient conditions for time-periodic bifurcation to take place.
\end{abstract}

Keywords: fluid-structure interaction; Navier-Stokes equations; Hopf bifurcation; falling sphere

MSC: 35Q30; 76D05; 35B32; 76T99

\section{Introduction}

The motion of spheres falling or rising in a viscous liquid has long been recognized as a fundamental topic of research, not only for its intrinsic interest but also for its role in applied sciences; see [1-7] and the bibliography therein. Even though the dynamics may be different depending on whether the sphere is light (ascending) or heavy (falling) [8], its qualitative behavior is rather similar in both cases. More specifically, let $\rho_{\mathcal{S}}$ and $\rho_{\mathcal{L}}$ be the density of the sphere and of the liquid, respectively, and be denoted by $\lambda$ a suitable nondimensional number depending on $\left|\rho_{\mathcal{S}} / \rho_{\mathcal{L}}-1\right|$ (Galilei number); see (2). Then, experimental and numerical tests $[1,2,5,6]$ show that steady regimes occur as long as $\lambda$ is not too "large". Precisely, in a first range of Galilei numbers, the sphere merely translates (no spin) with constant translational velocity, $\tau_{0}$, parallel to the direction of gravity, $\boldsymbol{e}$. In this situation, the liquid flow is axisymmetric around $e$. For $\lambda$ above a first critical value, there is a breaking of symmetry from axisymmetry to planar symmetry. However, the motion is still translatory but now $\tau_{0}$ and $e$ are no longer parallel. If $\lambda$ is further increased to some higher critical value, $\lambda_{c}$ (say), then a second (Hopf) bifurcation occurs-the flow ceases to be steady and becomes instead purely time-periodic, with the constant translatory motion of the sphere giving way to an oscillating oblique movement. At even greater values of $\lambda$, a chaotic regime eventually sets in.

The objective of this paper is to furnish a rigorous mathematical contribution to the interpretation of some of the above bifurcation phenomena.

We recall that, from a strict mathematical viewpoint, the study of bifurcation, in fluid mechanics as in other branches of mathematical physics, presents a fundamental challenge. It consists in determining the appropriate functional setting where the problem can be formulated in order to be addressed by general abstract theory. In the situation at hand, this aspect is particularly intriguing, since the region of flow is unbounded in all directions, which implies that 0 is a point of the essential spectrum of the relevant linearized operator [9]. This is a crucial and well-known problem $[10,11]$ that prevents one from using classical 
approaches that are, instead, very successful in the case of bounded flow, where the above spectral issue is absent [12-14].

In the past few years, we have introduced a new, general approach to bifurcation that allows us to overcome the above problem and to provide sufficient (and necessary) conditions for the occurrence of bifurcation, in both steady-state and time-periodic cases, and for both bounded and unbounded flow $[15,16]$. The basic idea of this approach is to formulate the problem not in classical Sobolev spaces but, instead, in homogeneous Sobolev spaces, characterized by the property that the various derivatives involved may have different summability properties in the neighborhood of spatial infinity. In such a framework, the spectral issue mentioned earlier on is totally absent. In [16-18] we have employed this approach to study the bifurcation properties of a Navier-Stokes liquid past a fixed body, namely, when the body is kept in a given configuration by suitable forces and torques. More recently, we have used the new method to study steady-state bifurcation of a falling (or ascending) sphere in a viscous liquid under the action of gravity [19], which is a bona fide fluid-structure interaction problem. In particular, we have shown that a requirement for the occurrence of the above type of bifurcation with $\tau_{0}$ parallel to $\boldsymbol{e}$, is that the relevant linearized operator, defined in an appropriate function space, has 1 as a simple eigenvalue crossing the imaginary axis at "non-zero speed" (transversality condition). Remarkably, this requirement formally coincides with the classical generic bifurcation condition for a flow in a bounded domain [13].

In this article we continue and - to an extent—complete the research initiated in [19], by investigating the occurrence of time-periodic bifurcation of the coupled system sphereliquid under the action of gravity. More precisely, we show that, once the problem is formulated in the appropriate functional setting, we can obtain a bifurcation criterion along the lines of the classical Hopf theory. Namely, it suffices that the relevant linearized operator has a non-resonating, simple imaginary eigenvalue satisfying the transversality condition. In order to reach this goal, we follow [16,18] and split the unknowns into the sum of their average over a period plus an oscillatory component. In this way, the original problem transforms into a coupled system of nonlinear elliptic-parabolic equations. We then prove that such a system can be written as two coupled operator equations in suitable spaces, with the relevant operators satisfying all the assumptions of the abstract theory introduced in [16], which thus provides the desired bifurcation results.

The plan of the paper is as follows-in Section 2 we give the precise formulation of the problem and collect some standard notation. In Section 3, we present the abstract time-periodic bifurcation result proved in [16]; see Theorem 1. Successively, in Section 4, we recollect some fundamental function spaces introduced in $[20,21]$ and, for some of them, recall their relevant properties. The following Section 5 is dedicated to the existence of a unique steady-state solution branch parametrized in the Galilei number $\lambda$. To this end, we first show, in Theorem 2, that for any given $\lambda \neq 0$ there exists a corresponding steady-state solution, $s(\lambda)$, in a suitable homogeneous Sobolev space. Successively, we prove that if there is $\lambda_{c}$ such that the linearization, $\mathcal{L}$, around $s\left(\lambda_{c}\right)$ is trivial, then there exists a unique analytic family of steady-state solutions in a neighborhood of $\lambda_{c}$ where the translational velocity of the sphere is parallel to that of $s\left(\lambda_{c}\right)$; see Theorem 3. In Section 6, we investigate some important spectral properties of $\mathcal{L}$ in the Lebesgue space $L^{2}$. Precisely, we show that the intersection of the spectrum of $\mathcal{L}$ with the imaginary axis is constituted, at most, by a countable number of eigenvalues of finite multiplicity that can only cluster at 0 ; see Theorem 4. The main objective of Section 7 is to establish the Fredholm property of the time-periodic linearized operator in a suitable space of functions with zero average over a period. In particular, in Theorem 5 we prove that such operator is Fredholm of index 0 . With the help of the results established in the previous sections, in Section 8 we then secure that the original problem is written in an abstract setting where the general theory recalled in Section 4 applies. Therefore, thanks to Theorem 1, in Theorem 6 we give sufficient conditions for the existence of a time-periodic branch in the neighborhood of the steady-state solution $\mathrm{s}\left(\lambda_{\mathrm{c}}\right)$. As already mentioned, these conditions amount to the request 
that the operator $\mathcal{L}$, suitably defined, has a non-resonating, simple imaginary eigenvalue satisfying the transversality condition. In the final Section 9, we consider the problem of the motion of the sphere in the time-periodic regime. In this regard, we give necessary and sufficient conditions for the occurrence of a horizontal oscillation of the center of mass, in a neigborhood of the "critical" value $\lambda_{c}$; see Theorem 7.

\section{Formulation of the Problem}

A sphere, $\mathcal{S}$, of constant density, $\rho_{\mathcal{S}}$ and radius $R$ freely moves under the action of gravity in an otherwise quiescent Navier-Stokes liquid, $\mathcal{L}$, that fills the entire space outside $\mathcal{S}$. We assume that $\mathcal{S}$ is not floating, namely, it has a non-zero buoyancy. This means that, denoting by $\rho_{\mathcal{L}}$ the density of the liquid, we take $|\alpha|:=\left|\rho_{\mathcal{S}} / \rho_{\mathcal{L}}-1\right|>0$. Just to fix the ideas, we shall assume $\alpha>0$ (falling sphere). However, all results continue to hold in the case of positive buoyancy (rising sphere) by simply replacing $\alpha$ with $-\alpha$.

Let $\mathcal{F}=\left\{O, e_{1}, e_{2}, e_{3}\right\}$ be a frame with the origin at the center of $\mathcal{S}$ (三 center of mass of $\mathcal{S}$ ) and the axis $e_{1}$ oriented along the acceleration of gravity $g$. The dynamics of the coupled system $\mathcal{S} \cup \mathcal{L}$ in $\mathcal{F}$ are then governed by the following set of non-dimensional equations [20] (Section 4)

$$
\begin{aligned}
& \left.\begin{array}{l}
\partial_{t} \boldsymbol{v}+\lambda(\boldsymbol{v}-\boldsymbol{\tau}) \cdot \nabla \boldsymbol{v}=\Delta \boldsymbol{v}-\nabla p \\
\operatorname{div} \boldsymbol{v}=0
\end{array}\right\} \text { in } \Omega \times(0, \infty) \\
& \boldsymbol{v}=\boldsymbol{\tau}+\boldsymbol{\omega} \times \boldsymbol{x} \text { at } \partial \Omega \times(0, \infty), \lim _{|x| \rightarrow \infty} v(x, t)=\mathbf{0}, t \in(0, \infty), \\
& M \dot{\tau}+\int_{\partial \Omega} \mathbb{T}(v, p) \cdot \boldsymbol{n}=\lambda \boldsymbol{e}_{1}, \mathcal{I} \dot{\boldsymbol{\omega}}+\int_{\partial \Omega} \boldsymbol{x} \times \mathbb{T}(\boldsymbol{v}, p) \cdot \boldsymbol{n}=0 \text { in }(0, \infty) .
\end{aligned}
$$

Here, $\Omega=\mathbb{R}^{3} \backslash \Omega_{0}$, with $\Omega_{0}$ volume occupied by $\mathcal{S}$. Furthermore, $\boldsymbol{v}$ and $p+\boldsymbol{e}_{1} \cdot \boldsymbol{x}$ are (non-dimensional) velocity and pressure fields of $\mathcal{L}$, while $\tau, \omega$ stand for (non-dimensional) translational and angular velocities of $\mathcal{S}$. Moreover,

$$
\lambda=\sqrt{\alpha g R^{3}} / \nu(>0)
$$

is the (dimensionless) Galilei number, with $\nu$ kinematic viscosity of $\mathcal{L}$. Also, $M=4 \pi \rho_{\mathcal{S}} / 3 \rho_{\mathcal{L}}$ and $\mathcal{I}=8 \pi \rho_{\mathcal{S}} / 15 \rho_{\mathcal{L}}$ are non-dimensional mass and central moment of inertia of $\mathcal{S}$, and $n$ is the outer unit normal to $\partial \Omega$. Finally,

$$
\mathbb{T}(\boldsymbol{v}, p)=-p \mathbb{1}+2 \mathbb{D}(\boldsymbol{v}), \mathbb{D}(\boldsymbol{v}):=\frac{1}{2}\left(\nabla v+(\nabla v)^{\top}\right),
$$

is the Cauchy tensor with $\mathbb{1}$ identity tensor and $\top$ denoting transpose.

Of particular significance is the subclass of solutions to (1) constituted by those fields $\left(\boldsymbol{v}_{0}, p_{0}, \boldsymbol{\tau}_{0}, \omega_{0}\right)$ that are time independent, namely, they solve the following boundaryvalue problem

$$
\begin{gathered}
\left.\lambda\left(\boldsymbol{v}_{0}-\boldsymbol{\tau}_{0}\right) \cdot \nabla \boldsymbol{v}_{0}=\Delta \boldsymbol{v}_{0}-\nabla p_{0}\right) \text { in } \Omega \\
\operatorname{div} \boldsymbol{v}_{0}=0 \\
\boldsymbol{v}_{0}=\boldsymbol{\tau}_{0}+\boldsymbol{\omega}_{0} \times \boldsymbol{x} \text { at } \partial \Omega, \lim _{|x| \rightarrow \infty} \boldsymbol{v}_{0}(x)=\mathbf{0}, \\
\int_{\partial \Omega} \mathbb{T}\left(\boldsymbol{v}_{0}, p_{0}\right) \cdot \boldsymbol{n}=\lambda \boldsymbol{e}_{1}, \int_{\partial \Omega} \boldsymbol{x} \times \mathbb{T}\left(\boldsymbol{v}_{0}, p_{0}\right) \cdot \boldsymbol{n}=\mathbf{0} .
\end{gathered}
$$

Solutions $\left(v_{0}, p_{0}, \tau_{0}, \omega_{0}\right)$ to (3) describe the so called steady free falls of the sphere in the viscous liquid, and, as explained in the introductory section, their behavior depends on the parameter $\lambda$.

In mathematical terms, the time-periodic bifurcation problem can be formulated as follows-let $\lambda_{c}>0$, let $U=U\left(\lambda_{c}\right)$ be a neighborhood of $\lambda_{c}$, and let $s(\lambda):=\left(v_{0}, p_{0}, \tau_{0}, \omega_{0}\right)(\lambda)$, 
$\lambda \in U\left(\lambda_{\mathrm{c}}\right)$, be a sufficiently smooth family of solutions to (3). The objective is then to prove the existence of time-periodic solutions to (1) "around" $s\left(\lambda_{c}\right)$. Since the period $T:=2 \pi / \zeta$ of such solutions is unknown, it is customary to scale the time by introducing the new variable $\mathrm{s}=\zeta t$. Therefore, writing

$\boldsymbol{v}(x, t)=\boldsymbol{u}(x, t)+\boldsymbol{v}_{0}(x), p(x, t)=\mathrm{p}(x, t)+p_{0}(x), \boldsymbol{\tau}(t)=\gamma(t)+\boldsymbol{\tau}_{0}, \boldsymbol{\omega}(t)=\boldsymbol{\omega}(t)+\boldsymbol{\omega}_{0}$,

our bifurcation problem means that we must find a $2 \pi$-periodic solution-branch $(\boldsymbol{u}, \mathrm{p}, \boldsymbol{\gamma}, \boldsymbol{\omega})(\lambda)$, $\lambda \in U\left(\lambda_{\mathrm{c}}\right)$, to the following set of equations

$$
\begin{gathered}
\left.\begin{array}{c}
\zeta \partial_{\mathrm{s}} \boldsymbol{u}-\lambda \boldsymbol{\tau}_{0} \cdot \nabla \boldsymbol{u}+\lambda\left(\boldsymbol{v}_{0} \cdot \nabla \boldsymbol{u}+(\boldsymbol{u}-\gamma) \cdot \nabla \boldsymbol{v}_{0}\right)+\lambda(\boldsymbol{u}-\gamma) \cdot \nabla \boldsymbol{u}=\Delta \boldsymbol{u}-\nabla \mathrm{p} \\
\operatorname{div} \boldsymbol{u}=0
\end{array}\right\} \text { in } \Omega \times \mathbb{R}, \\
\boldsymbol{u}=\gamma+\boldsymbol{\omega} \times \boldsymbol{x} \text { at } \partial \Omega \times \mathbb{R}, \lim _{|\boldsymbol{x}| \rightarrow \infty} \boldsymbol{u}(x, t)=\mathbf{0}, \quad t \in \mathbb{R}, \\
M \dot{\gamma}+\int_{\partial \Omega} \mathbb{T}(\boldsymbol{u}, \mathrm{p}) \cdot \boldsymbol{n}=\mathbf{0}, \mathcal{I} \dot{\boldsymbol{\omega}}+\int_{\partial \Omega} \boldsymbol{x} \times \mathbb{T}(\boldsymbol{u}, \mathrm{p}) \cdot \boldsymbol{n}=\mathbf{0} \text { in } \mathbb{R} .
\end{gathered}
$$

Our strategy to solve this problem consists in rewriting (4) as operator equations in suitable Banach spaces, with the involved operators satisfying a certain number of fundamental properties. Once this goal is accomplished, we will be able to employ the general theory introduced in [16] and recalled in Section 3 and derive sufficient conditions for the existence of a time-periodic bifurcating branch.

Before performing our study, we recall the main notation used in the paper. With the origin at the center of $\Omega_{0}$, we set $B_{R}:=\{|x|<R\}$, and, for $R>1=\operatorname{diam} \Omega_{0}$, $\Omega_{R}:=\Omega \cap B_{R}, \Omega^{R}:=\Omega \cap\{|x|>R\}$. As customary, for a domain $A \subseteq \mathbb{R}^{3}, L^{q}=L^{q}(A)$ is the Lebesgue space with norm $\|\cdot\|_{q, A}$, and $W^{m, q}=W^{m, q}(A)$ denotes Sobolev space, $m \in \mathbb{N}, q \in[1, \infty]$, with norm $\|\cdot\|_{m, q, A}$. If $u \in L^{q}(A), v \in L^{q^{\prime}}(A), q^{\prime}=q /(q-1)$, we set $(u, v)_{A}=\int_{A} u v$. Furthermore, $D^{m, q}=D^{m, q}(A)$ are homogeneous Sobolev spaces with semi-norm $|u|_{m, q, A}:=\sum_{|l|=m}\left\|D^{l} u\right\|_{q}$. In the above notation, the subscript " $A$ " will be generally omitted, unless confusion arises. A function $u: A \times \mathbb{R} \mapsto \mathbb{R}^{3}$ is $2 \pi$-periodic, if $u(\cdot, \mathrm{s}+2 \pi)=u(\cdot \mathrm{s})$, for a.a. $\mathrm{s} \in \mathbb{R}$, and we set $\bar{u}:=\frac{1}{2 \pi} \int_{0}^{2 \pi} u(t) \mathrm{d} t$. If $B$ is a semi-normed real Banach space with semi-norm $\|\cdot\|_{B}, r=[1, \infty]$, we denote by $L^{r}(0,2 \pi ; B)$ the class of functions $u:(0,2 \pi) \rightarrow B$ such that

$$
\|u\|_{L^{r}(B)} \equiv\left\{\begin{array}{l}
\left(\int_{0}^{2 \pi}\|u(t)\|_{B}^{r} \mathrm{~d} t\right)^{\frac{1}{r}}<\infty, \text { if } r \in[1, \infty) \\
\text { ess sup }\|u(t)\|_{B}<\infty, \text { if } r=\infty .
\end{array}\right.
$$

Furthermore, we define

$$
W^{1,2}(0,2 \pi ; B)=\left\{u \in L^{2}(0,2 \pi ; B): \partial_{t} u \in L^{2}(0,2 \pi ; B),\right\} .
$$

Unless otherwise stated, we shall write $L^{r}(B)$ for $L^{r}(0,2 \pi ; B)$, and so forth. By $B_{\mathbb{C}}:=$ $B+\mathrm{i} B$ we denote the complexification of $B$. If $M$ is a map between two spaces, $\mathrm{D}[M], \mathrm{N}[M]$, $\mathrm{R}[M]$ and $\mathrm{P}[M]$ will indicate its domain, null space, range, and resolvent set, respectively. Finally, by $c, c_{0}, c_{1}$, and so forth, we denote positive constants, whose particular value is unessential to the context. When we wish to emphasize the dependence of $c$ on some parameter $\mu$, we shall write $c(\mu)$ or $c_{\mu}$.

\section{An Abstract Bifurcation Theorem}

Objective of this section is to recall a time-periodic bifurcation theorem for a general class of operator equations proved in [16]. Before stating the result, however, we first would like to make some comments that will also provide the motivation of this approach. 
Many evolution problems in mathematical physics can be formally written in the form

$$
u_{t}+L(u)=N(u, \mu) \text {, }
$$

where $L$ is a linear differential operator (with appropriate homogeneous boundary conditions), and $N$ is a nonlinear operator depending on the parameter $\mu \in \mathbb{R}$, such that $N(0, \mu)=0$ for all admissible values of $\mu$. Then, roughly speaking, time-periodic bifurcation for (5) amounts to show the existence a family of non-trivial time-periodic solutions $u=u(\mu ; t)$ of (unknown) period $T=T(\mu)$ (T-periodic solutions) in a neighborhood of $\mu=0$, and such that $u\left(\mu_{;} \cdot\right) \rightarrow 0$ as $\mu \rightarrow 0$. Setting $\mathrm{s}:=2 \pi t / T \equiv \zeta t,(71)$ becomes

$$
\zeta u_{\mathrm{s}}+L(u)=N(u, \mu)
$$

and the problem reduces to find a family of $2 \pi$-periodic solutions to (6) with the above properties. We now write $u=\bar{u}+(u-\bar{u}):=v+w$ and observe that (6) is formally equivalent to the following two equations

$$
\begin{aligned}
& L(v)=\overline{N(v+w, \mu)}:=N_{1}(v, w, \mu) \\
& \zeta w_{\mathrm{s}}+L(w)=N(v+w, \mu)-\overline{N(v+w, \mu)}:=N_{2}(v, w, \mu) .
\end{aligned}
$$

At this point, the crucial issue is that in many applications -typically when the physical system evolves in an unbounded spatial region- the "steady-state component" $v$ lives in function spaces with quite less "regularity" (here, 'regularity' is meant in the sense of behavior at large spatial distances.) than the space where the "oscillatory" component $w$ does. For this reason, it is much more appropriate to study the two equations in (7) in two different function classes. As a consequence, even though formally being the same as differential operators, the operator $L$ in $(7)_{1}$ acts on and ranges into spaces different than those the operator $L$ in $(7)_{2}$ does. With this in mind, (7) becomes

$$
L_{1}(v)=N_{1}(v, w, \mu) ; \zeta w_{\mathrm{s}}+L_{2}(w)=N_{2}(v, w, \mu) .
$$

The general abstract theory that we are about to describe stems exactly from the above considerations.

Let $\mathcal{X}, \mathcal{Y}$, be (real) Banach spaces with norms $\|\cdot\|_{\mathcal{X}},\|\cdot\|_{\mathcal{Y}}$, respectively, and let $\mathcal{Z}$ be a (real) Hilbert space with norm $\|\cdot\|_{\mathcal{Z}}$ and corresponding scalar product $\langle\cdot, \cdot\rangle$. Moreover, denote by

$$
L_{1}: \mathcal{X} \mapsto \mathcal{Y}
$$

a bounded linear operator, and by

$$
L_{2}: \mathrm{D}\left[L_{2}\right] \subset \mathcal{Z} \mapsto \mathcal{Z}
$$

a densely defined, closed linear operator, with a non-empty resolvent set $\mathrm{P}\left(L_{2}\right)$. For a fixed (once and for all) $\theta \in \mathrm{P}\left(L_{2}\right)$ we denote by $\mathcal{W}$ the linear subspace of $\mathcal{Z}$ closed under the norm $\|w\|_{\mathcal{W}}:=\left\|\left(L_{2}+\theta I\right) w\right\|_{\mathcal{Z}}$, where $I$ stands for the identity operator. We also define the following spaces

$$
\begin{aligned}
\mathcal{Z}_{2 \pi, 0} & :=\left\{w \in L^{2}(0,2 \pi ; \mathcal{Z}): 2 \pi \text {-periodic with } \bar{w}=0\right\} \\
\mathcal{W}_{2 \pi, 0} & :=\left\{w \in L^{2}(0,2 \pi ; \mathcal{Z}), w_{t} \in L^{2}(0,2 \pi ; \mathcal{Z}): 2 \pi \text {-periodic with } \bar{w}=0\right\} .
\end{aligned}
$$

Next, let

$$
N: \mathcal{X} \times \mathcal{W}_{2 \pi, 0} \times \mathbb{R} \mapsto \mathcal{Y} \oplus \mathcal{H}_{2 \pi, 0}
$$


be a (nonlinear) map satisfying the following properties:

$$
\begin{aligned}
& N_{1}:(v, w, \mu) \in \mathcal{X} \times \mathcal{W}_{2 \pi, 0} \times \mathbb{R} \mapsto \overline{N(v, w, \mu)} \in \mathcal{Y} \\
& N_{2}:=N-N_{1}: \mathcal{X} \times \mathcal{W}_{2 \pi, 0} \times \mathbb{R} \mapsto \mathcal{Z}_{2 \pi, 0} .
\end{aligned}
$$

The bifurcation problem can be then rigorously formulated as follows.

Bifurcation Problem: Find a neighborhood of the origin $U(0,0,0) \subset \mathcal{X} \times \mathcal{W}_{2 \pi, 0} \times \mathbb{R}$ such that the equations

$$
L_{1}(v)=N_{1}(v, w, \mu), \text { in } \mathcal{Y} ; \zeta w_{\mathrm{s}}+L_{2}(w)=N_{2}(v, w, \mu), \text { in } \mathcal{Z}_{2 \pi, 0},
$$

possess there a family of non-trivial $2 \pi$-periodic solutions $(v(\mu), w(\mu ; \tau))$ for some $\omega=\omega(\mu)>0$, such that $(v(\mu), w(\mu ; \cdot)) \rightarrow 0$ in $\mathcal{X} \times \mathcal{W}_{2 \pi, 0}$ as $\mu \rightarrow 0$.

Whenever the Bifurcation Problem admits a positive answer, we say that $(u=0, \mu=0)$ is a bifurcation point. Moreover, the bifurcation is called supercritical [resp. subcritical] if the family of solutions $(v(\mu), w(\mu ; \tau))$ exists only for $\mu>0$ [resp. $\mu<0$ ].

In order to provide sufficient conditions for the resolution of the above problem, we need the following assumptions (H1)-(H4) on the involved operators.

Hypothesis 1. $L_{1}$ is a homeomorphism;

Hypothesis 2. There exists $v_{0}:=\mathrm{i} \zeta_{0}, \zeta_{0}>0$ such that $L_{2}-v_{0} I$ is Fredholm of index 0 , and $\operatorname{dim} \mathrm{N}_{\mathbb{C}}\left[L_{2}-v_{0} I\right]=1$ with $\mathrm{N}_{\mathbb{C}}\left[L_{2}-v_{0} I\right] \cap \mathrm{R}_{\mathbb{C}}\left[L_{2}-v_{0} I\right]=\{0\}$. Namely, $v_{0}$ is a simple eigenvalue of $L_{2}$. Moreover, $k v_{0} \in \mathrm{P}\left(L_{2}\right)$, for all $k \in \mathbb{N} \backslash\{0,1\}$.

Hypothesis 3. The operator

$$
\mathcal{Q}: w \in \mathcal{W}_{2 \pi, 0} \mapsto \zeta_{0} w_{\mathrm{s}}+L_{2}(w) \in \mathcal{Z}_{2 \pi, 0}
$$

is Fredholm of index 0;

Hypothesis 4. The nonlinear operators $N_{1}, N_{2}$ are analytic in the neighborhood $U_{1}(0,0,0) \subset$ $\mathcal{X} \times \mathcal{W}_{2 \pi, 0} \times \mathbb{R}$, namely, there exists $\delta>0$ such that for all $(v, w, \mu)$ with $\|v\|_{\mathcal{X}}+\|w\|_{\mathcal{W}_{2 \pi, 0}}+$ $|\mu|<\delta$, the Taylor series

$$
\begin{aligned}
& N_{1}(v, w, \mu)=\sum_{k, l, m=0}^{\infty} R_{k l m} v^{k} w^{l} \mu^{m}, \\
& N_{2}(v, w, \mu)=\sum_{k, l, m=0}^{\infty} S_{k l m} v^{k} w^{l} \mu^{m},
\end{aligned}
$$

are absolutely convergent in $\mathcal{Y}$ and $\mathcal{Z}_{2 \pi, 0}$, respectively, for all $(v, w, \mu) \in U_{1}$. Moreover, we assume that the multi-linear operators $R_{k l m}$ and $S_{k l m}$ satisfy $R_{k l m}=S_{k l m}=0$ whenever $k+l+m \leq 1$, and $R_{011}=R_{00 m}=S_{00 m}=0$, all $m \geq 2$.

Before stating the bifurcation result, we need to recall a relevant consequence of the above assumptions. Let

$$
L_{2}(\mu):=L_{2}+\mu S_{011}
$$

and observe that, by $(\mathrm{H} 2), v_{0}$ is a simple eigenvalue of $L_{2}(0) \equiv L_{2}$. Therefore, denoting by $v(\mu)$ the eigenvalues of $L_{2}(\mu)$, it follows (e.g., [14] (Proposition 79.15 and Corollary 79.16)) that in a neighborhood of $\mu=0$ the map $\mu \mapsto v(\mu)$ is well defined and of class $C^{\infty}$. We may then state the following bifurcation result, whose proof is given in [16] (Theorem 3.1). 
Theorem 1. Suppose (H1)-(H4) hold and, in addition,

$$
\Re\left[v^{\prime}(0)\right] \neq 0,
$$

namely, the eigenvalue $v(\mu)$ crosses the imaginary axis with "non-zero speed". Moreover, let $v_{0}$ be a normalized eigenvector of $L_{2}$ corresponding to the eigenvalue $v_{0}$, and set $v_{1}:=\Re\left[v_{0} \mathrm{e}^{-\mathrm{is}}\right]$. Then, the following properties are valid.

(a) Existence. There are analytic families

$$
(v(\varepsilon), w(\varepsilon), \zeta(\varepsilon), \mu(\varepsilon)) \in \mathcal{X} \times \mathcal{W}_{2 \pi, 0} \times \mathbb{R}_{+} \times \mathbb{R}
$$

satisfying (9), for all real $\varepsilon$ in a neighborhood $\mathcal{I}(0)$ of 0 , and such that

$$
\left(v(\varepsilon), w(\varepsilon)-\varepsilon v_{1}, \zeta(\varepsilon), \mu(\varepsilon)\right) \rightarrow\left(0,0, \zeta_{0}, 0\right) \text { as } \varepsilon \rightarrow 0 .
$$

(b) Uniqueness. There is a neighborhood

$$
U\left(0,0, \zeta_{0}, 0\right) \subset \mathcal{X} \times \mathcal{W}_{2 \pi, 0} \times \mathbb{R}_{+} \times \mathbb{R}
$$

such that every (nontrivial) $2 \pi$-periodic solution to (9), lying in $U$ must coincide, up to a phase shift, with a member of the family (10).

(c) Parity. The functions $\zeta(\varepsilon)$ and $\mu(\varepsilon)$ are even:

$$
\zeta(\varepsilon)=\zeta(-\varepsilon), \mu(\varepsilon)=\mu(-\varepsilon), \text { for all } \varepsilon \in \mathcal{I}(0) .
$$

Consequently, the bifurcation due to these solutions is either subcritical or supercritical, a two-sided bifurcation being excluded (Unless $\mu \equiv 0$ ).

\section{Relevant Function Spaces and Related Properties}

In this section we will introduce certain function classes along with some of their most important properties.

Let $\mathcal{R}$ be the class of velocity fields in a rigid motion:

$$
\boldsymbol{R}=\left\{\hat{\boldsymbol{u}} \in C^{\infty}\left(\mathbb{R}^{3}\right): \hat{\boldsymbol{u}}=\hat{\boldsymbol{u}}_{1}+\hat{\boldsymbol{u}}_{2} \times x, \text { for some } \hat{\boldsymbol{u}}_{1}, \hat{\boldsymbol{u}}_{2} \in \mathbb{R}^{3}\right\},
$$

and set

$$
\begin{aligned}
& \mathcal{K}=\mathcal{K}\left(\mathbb{R}^{3}\right)=\left\{\boldsymbol{\varphi} \in C_{0}^{\infty}\left(\overline{\mathbb{R}^{3}}\right): \text { in } \mathbb{R}^{3} ; \boldsymbol{\varphi}(x)=\hat{\boldsymbol{\varphi}}, \text { some } \hat{\boldsymbol{\varphi}} \in \mathcal{R} \text { in a neighborhood of } \Omega_{0}\right\} \\
& \mathcal{C}=\mathcal{C}\left(\mathbb{R}^{3}\right)=\left\{\boldsymbol{\varphi} \in \mathcal{K}\left(\mathbb{R}^{3}\right): \operatorname{div} \boldsymbol{\varphi}=0 \text { in } \mathbb{R}^{3}\right\} .
\end{aligned}
$$

We shall call $\hat{\varphi}_{1}, \hat{\varphi}_{2}$ the characteristic vectors of the function $\varphi$. In $\mathcal{K}$ we introduce the scalar product

$$
M \hat{\boldsymbol{\varphi}}_{1} \cdot \hat{\psi}_{1}+\mathcal{I} \hat{\boldsymbol{\varphi}}_{2} \cdot \hat{\psi}_{2}+(\boldsymbol{\varphi}, \psi)_{\Omega}, \boldsymbol{\varphi}, \psi \in \mathcal{K},
$$

and define the following spaces (Even though $\boldsymbol{x} \times \boldsymbol{n}=\mathbf{0}$ if $x \in \partial \Omega$, for notational convenience we will keep the term $\hat{h}_{2}$.)

$$
\begin{array}{r}
\mathcal{L}^{2}=\mathcal{L}^{2}\left(\mathbb{R}^{3}\right):=\left\{\text { completion of } \mathcal{K}\left(\mathbb{R}^{3}\right) \text { in the norm induced by }(12)\right\}, \\
\mathcal{H}=\mathcal{H}\left(\mathbb{R}^{3}\right):=\left\{\text { completion of } \mathcal{C}\left(\mathbb{R}^{3}\right) \text { in the norm induced by }(12)\right\} \\
\mathcal{G}=\mathcal{G}\left(\mathbb{R}^{3}\right):=\left\{\boldsymbol{h} \in \mathcal{L}^{2}\left(\mathbb{R}^{3}\right): \text { there is } p \in D^{1,2}(\Omega) \text { such that } \boldsymbol{h}=\nabla p \text { in } \Omega,\right. \\
\left.\quad \text { and } \boldsymbol{h}=-M^{-1} \int_{\partial \Omega} p \boldsymbol{n}-\mathcal{I}^{-1}\left(\int_{\partial \Omega} p \boldsymbol{y} \times \boldsymbol{n}\right) \times x \text { in } \Omega_{0}\right\} .
\end{array}
$$

In [21] (Theorem 3.1 and Lemma 3.2) the following characterization of the spaces $\mathcal{L}^{2}$ and $\mathcal{H}$ is proved. 
Lemma 1. Let $\Omega$ be Lipschitz. Then

$$
\begin{aligned}
& \mathcal{L}^{2}\left(\mathbb{R}^{3}\right)=\left\{\boldsymbol{u} \in L^{2}\left(\mathbb{R}^{3}\right): \boldsymbol{u}=\hat{\boldsymbol{u}} \text { in } \Omega_{0}, \text { for some } \hat{\boldsymbol{u}} \in \boldsymbol{R}\right\} \\
& \mathcal{H}\left(\mathbb{R}^{3}\right)=\left\{\boldsymbol{u} \in \mathcal{L}^{2}\left(\mathbb{R}^{3}\right): \operatorname{div} \boldsymbol{u}=0\right\} .
\end{aligned}
$$

Furthermore, by an argument entirely analogous to that employed in [21] (Theorem 3.2) one shows:

Lemma 2. The following orthogonal decomposition holds

$$
\mathcal{L}^{2}\left(\mathbb{R}^{3}\right)=\mathcal{H}\left(\mathbb{R}^{3}\right) \oplus \mathcal{G}\left(\mathbb{R}^{3}\right) .
$$

We next introduce the space

$$
\mathcal{V}=\mathcal{V}\left(\mathbb{R}^{3}\right) \equiv\left\{\text { completion of } \mathcal{C}\left(\mathbb{R}^{3}\right) \text { in the norm }\|\mathbb{D}(\cdot)\|_{2}\right\} .
$$

The basic properties of the space $\mathcal{V}$ are collected in the next lemma, whose proof is given in [20] (Lemmas 9-11).

Lemma 3. $\mathcal{V}$ is a Hilbert space endowed with the scalar product

$$
[u, w]=\int_{\Omega} \mathbb{D}(u): \mathbb{D}(w), u, w \in \mathcal{V} .
$$

Furthermore, the following characterization holds

$$
\mathcal{V}=\left\{\boldsymbol{u} \in L^{6}\left(\mathbb{R}^{3}\right) \cap D^{1,2}\left(\mathbb{R}^{3}\right) ; \operatorname{div} \boldsymbol{u}=0 \text { in } \mathbb{R}^{3} ; \boldsymbol{u}(y)=\hat{\boldsymbol{u}}, y \in \Omega_{0} \text {, some } \hat{\boldsymbol{u}} \in \boldsymbol{R}\right\} \text {. }
$$

Also, for each $\boldsymbol{u} \in \mathcal{V}$, we have

$$
\|\nabla \boldsymbol{u}\|_{2}=\sqrt{2}\|\mathbb{D}(\boldsymbol{u})\|_{2},
$$

and

$$
\|\boldsymbol{u}\|_{6} \leq \kappa_{0}\|\mathbb{D}(\boldsymbol{u})\|_{2}, \boldsymbol{u} \in \mathcal{V},
$$

for some numerical constant $\kappa_{0}>0$ (Recall that, in our non-dimensionalization, the sphere has radius 1$)$. Finally, there is another positive numerical constant $\kappa_{1}$ such that

$$
\left|\hat{\boldsymbol{u}}_{1}\right|+\left|\hat{\boldsymbol{u}}_{2}\right| \leq \kappa_{1}\|\mathbb{D}(\boldsymbol{u})\|_{2} .
$$

Let $\mathcal{V}^{-1}=\mathcal{V}^{-1}\left(\mathbb{R}^{3}\right)$ be the dual space of $\mathcal{V}$, and let $\langle\cdot, \cdot\rangle$ and $\|\cdot\|_{-1}$ be the corresponding duality pair and associated norm, respectively. Denote by $\boldsymbol{e} \in \mathbb{R}^{3}$ a given unit vector and consider the space

$$
X_{\boldsymbol{e}}\left(\mathbb{R}^{3}\right):=\left\{\boldsymbol{u} \in \mathcal{V}\left(\mathbb{R}^{3}\right): \boldsymbol{e} \cdot \nabla \boldsymbol{u} \in \mathcal{V}^{-1}\right\}
$$

where $\boldsymbol{e} \cdot \nabla \boldsymbol{u} \in \mathcal{V}^{-1}$ means that there is $C=C(\boldsymbol{u})>0$ such that

$$
|(\boldsymbol{e} \cdot \nabla \boldsymbol{u}, \boldsymbol{\varphi})| \leq \mathcal{C}\|\mathbb{D}(\boldsymbol{\varphi})\|_{2}, \text { for all } \boldsymbol{\varphi} \in \mathcal{C}\left(\mathbb{R}^{3}\right) .
$$

Actually, from (19), the density of $\mathcal{C}$ in $\mathcal{V}$, and the Hahn-Banach theorem it follows that $\boldsymbol{e} \cdot \nabla \boldsymbol{u}$ can be uniquely extended to a bounded linear functional on the whole of $\mathcal{V}$, with

$$
\|\boldsymbol{e} \cdot \nabla \boldsymbol{u}\|_{-1}:=\sup _{\boldsymbol{\varphi} \in \mathcal{C} ;\|\mathbb{D}(\boldsymbol{\varphi})\|_{2}=1}|(\boldsymbol{e} \cdot \nabla \boldsymbol{u}, \boldsymbol{\varphi})| .
$$

Obviously, the functional

$$
\|\boldsymbol{u}\|_{X_{\boldsymbol{e}}}:=\|\mathbb{D}(\boldsymbol{u})\|_{2}+\left\|\partial_{1} \boldsymbol{u}\right\|_{-1},
$$


defines a norm in $X\left(\mathbb{R}^{3}\right)$.

In the following lemma, we collect the relevant properties of the space $X\left(\mathbb{R}^{3}\right)$. Their proofs are entirely analogous to [17] (Proposition 65) and [19] (Lemma 2.1), and therefore will be omitted.

Lemma 4. The space $X_{\boldsymbol{e}}\left(\mathbb{R}^{3}\right)$ endowed with the norm $\|\cdot\| x_{\boldsymbol{e}}$ is a reflexive, separable Banach space, dense in $\mathcal{V}\left(\mathbb{R}^{3}\right)$. Moreover, $X_{\boldsymbol{e}}\left(\mathbb{R}^{3}\right)$ is continuously embedded in $L^{4}\left(\mathbb{R}^{3}\right)$, and there is $c>0$ such that

$$
\|\boldsymbol{u}\|_{4} \leq c\left(\|\boldsymbol{e} \cdot \nabla \boldsymbol{u}\|_{-1}^{\frac{1}{4}}\|\mathbb{D}(\boldsymbol{u})\|_{2}^{\frac{3}{4}}+\|\mathbb{D}(\boldsymbol{u})\|_{2}\right) .
$$

Finally, we have

$$
\langle\boldsymbol{e} \cdot \nabla \boldsymbol{u}, \boldsymbol{u}\rangle=0, \text { for all } \boldsymbol{u} \in X_{\boldsymbol{e}}\left(\mathbb{R}^{3}\right)
$$

We next introduce the following spaces of time-periodic functions:

$$
\begin{aligned}
& L_{\sharp}^{2}=\left\{\boldsymbol{F} \in L^{2}(0,2 \pi) ; \boldsymbol{F} \text { is } 2 \pi \text {-periodic with } \overline{\boldsymbol{F}}=0\right\} \\
& W_{\sharp}^{1,2}=\left\{\chi \in L_{\sharp}^{2}(0,2 \pi) ; \dot{\chi} \in L^{2}(0,2 \pi)\right\} \\
& \mathcal{L}_{\sharp}^{2}:=\left\{\boldsymbol{f} \in L^{2}\left(L^{2}\right) ; \boldsymbol{f} \text { is } 2 \pi \text {-periodic, with } \overline{\boldsymbol{f}}=\mathbf{0}\right\} \\
& \mathcal{W}_{\sharp}^{2}:=\left\{\boldsymbol{u} \in W^{1,2}\left(L^{2}\right) \cap L^{2}\left(W^{2,2} \cap \mathcal{V}\right) ; \boldsymbol{u} \text { is } 2 \pi \text {-periodic, with } \overline{\boldsymbol{u}}=\mathbf{0}\right\},
\end{aligned}
$$

endowed with natural norms

$$
\begin{aligned}
& \|\chi\|_{L_{\sharp}^{2}}:=\|\chi\|_{L^{2}} ; \quad\|\chi\|_{W_{\sharp}^{1,2}}:=\|\chi\|_{W^{1,2}} \equiv\|\dot{\chi}\|_{L^{2}}+\|\chi\|_{L^{2}}, \\
& \|\boldsymbol{u}\|_{\mathcal{L}_{\sharp}^{2}}:=\|\boldsymbol{u}\|_{L^{2}\left(L^{2}\right)} ; \quad\|\boldsymbol{u}\|_{\mathcal{W}_{\sharp}^{2}}:=\|\boldsymbol{u}\|_{W^{1,2}\left(L^{2}\right)}+\|\boldsymbol{u}\|_{L^{2}\left(W^{2,2}\right)} .
\end{aligned}
$$

Finally, we set

$$
\mathcal{P}^{1,2}:=L^{2}\left(D^{1,2}\right)
$$

\section{On the Existence of a Steady-State Branch}

The goal of this section is two-fold. On the one hand, to establish existence of solutions to (3) in suitable function classes for all values of $\lambda>0$ and, on the other hand, to furnish sufficient conditions for the existence of a smooth solution branch around the "critical" value $\lambda_{c}$.

We begin to give the definition of weak solution.

Definition 1. A field $v: \mathbb{R}^{3} \mapsto \mathbb{R}^{3}$ is a weak solution to (3) if the following conditions hold.

(i) $\boldsymbol{v} \in \mathcal{V}\left(\mathbb{R}^{3}\right)$, and $\boldsymbol{\tau} \equiv \hat{\boldsymbol{v}}_{1} \neq \mathbf{0}$;

(ii) $v$ satisfies the relation: (For simplicity, we omit the subscript " 0 ".)

$$
2[\boldsymbol{v}, \boldsymbol{\varphi}]-\lambda \hat{\boldsymbol{\varphi}}_{1} \cdot \boldsymbol{e}_{1}=\lambda((\boldsymbol{\tau}-\boldsymbol{v}) \cdot \nabla \boldsymbol{v}, \boldsymbol{\varphi}), \text { for all } \boldsymbol{\varphi} \in \mathcal{C} .
$$

Remark 1. It is easy to show that, if a weak solution $v$ is sufficiently smooth in $\Omega$, then there exists a likewise smooth pressure field $p: \Omega \mapsto \mathbb{R}$ such that the quadruple $\left(\boldsymbol{v}, p, \tau \equiv \hat{\boldsymbol{v}}_{1}, \omega \equiv \hat{\boldsymbol{v}}_{2}\right)$ is a smooth solution to (3). To this end, we begin to obseve that, clearly, the validity of $(3)_{2,3}$ comes from the fact that $v \in \mathcal{V}\left(\mathbb{R}^{3}\right)$. If we integrate by parts the first term on the left-hand side of (21), we get

$$
2[v, \varphi]=-\int_{\Omega} \Delta v \cdot \boldsymbol{\varphi}+\hat{\varphi}_{1} \cdot \int_{\partial \Omega} 2 \mathbb{D}(v) \cdot n+\hat{\varphi}_{2} \cdot \int_{\partial \Omega} x \times(2 \mathbb{D}(v) \cdot n) .
$$

Thus, by taking, in particular, $\varphi \in \mathcal{D}(\Omega):=\left\{\boldsymbol{\varphi} \in C_{0}^{\infty}(\Omega): \operatorname{div} \boldsymbol{\varphi}=0\right\}$, from (21) and (22) we get

$$
\int_{\Omega}(-\Delta v-\lambda(\tau-v) \cdot \nabla v) \cdot \boldsymbol{\varphi}=0, \text { for all } \boldsymbol{\varphi} \in \mathcal{D}(\Omega),
$$


which, by well-known results, implies the existence of a (smooth) scalar field $p$ for which (3) ${ }_{1}$ holds. Now, using (3) $)_{1}$ and (22) we deduce

$\hat{\boldsymbol{\varphi}}_{1} \cdot\left(-\lambda \boldsymbol{e}_{1}+\int_{\partial \Omega} 2 \mathbb{D}(\boldsymbol{v}) \cdot \boldsymbol{n}\right)+\hat{\boldsymbol{\varphi}}_{2} \cdot \int_{\partial \Omega} \boldsymbol{x} \times(2 \mathbb{D}(\boldsymbol{v}) \cdot \boldsymbol{n})-\int_{\Omega} \nabla p \cdot \boldsymbol{\varphi}=\lambda \int_{\Omega_{0}}(\boldsymbol{\tau}-\boldsymbol{v}) \cdot \nabla \boldsymbol{v} \cdot \hat{\boldsymbol{\varphi}}$,

which, after an integration by parts leads to

$$
\hat{\boldsymbol{\varphi}}_{1} \cdot\left(-\lambda \boldsymbol{e}_{1}+\int_{\partial \Omega} \mathbb{T}(\boldsymbol{v}, p) \cdot \boldsymbol{n}\right)+\hat{\boldsymbol{\varphi}}_{2} \cdot \int_{\partial \Omega} \boldsymbol{x} \times \mathbb{T}(\boldsymbol{v}, p) \cdot \boldsymbol{n}=\lambda \int_{\Omega_{0}}(\boldsymbol{\tau}-\boldsymbol{v}) \cdot \nabla \boldsymbol{v} \cdot \hat{\boldsymbol{\varphi}}
$$

Recalling that, in $\Omega_{0}, \boldsymbol{v}, \varphi \in \boldsymbol{R}$, we get $\left(\boldsymbol{\tau} \equiv \hat{\boldsymbol{v}}_{1}\right)$

$$
\begin{aligned}
\int_{\Omega_{0}}(\tau-\boldsymbol{v}) \cdot \nabla \boldsymbol{v} \cdot \hat{\boldsymbol{\varphi}} & =\int_{\Omega_{0}}\left(\hat{\boldsymbol{v}}_{2} \times \boldsymbol{x}\right) \cdot \nabla\left(\hat{\boldsymbol{v}}_{2} \times \boldsymbol{x}\right) \cdot \hat{\boldsymbol{\varphi}} \\
& =\int_{\partial \Omega} \hat{\boldsymbol{v}}_{2} \times \boldsymbol{x} \cdot \boldsymbol{n}\left(\hat{\boldsymbol{v}}_{2} \times \boldsymbol{x} \cdot \hat{\boldsymbol{\varphi}}\right)-\int_{\Omega}\left(\hat{\boldsymbol{v}}_{2} \times \boldsymbol{x}\right) \cdot \mathbb{D}(\hat{\boldsymbol{\varphi}}) \cdot\left(\hat{\boldsymbol{v}}_{2} \times \boldsymbol{x}\right)=0
\end{aligned}
$$

because $\mathbb{D}(\hat{\boldsymbol{\varphi}})=0$, and $\boldsymbol{x} \times \boldsymbol{n}=\mathbf{0}$ for all $x \in \partial \Omega$. From the latter, (23), and the arbitrariness of $\hat{\varphi}$ we then conclude that a smooth weak solution $v$ and the associated pressure field $p$ satisfy also (3) 5,6 . Finally, as a consequence of the next lemma, we shall see that $v$ obeys also the asymptotic condition (3) 4 .

The next lemma establishes some important properties of weak solutions.

Lemma 5. Let $v$ be a weak solution to (3). Then, the following properties hold.

(a) $v \in C^{\infty}\left(\bar{\Omega}_{R}\right) \cap C^{\infty}(\Omega)$, all $R>1$, and there is a pressure field $p \in C^{\infty}\left(\bar{\Omega}_{R}\right) \cap C^{\infty}(\Omega)$, all $R>1$, such that the quadruple $\left(\boldsymbol{v}, p, \tau \equiv \hat{\boldsymbol{v}}_{1}, \boldsymbol{\omega} \equiv \hat{\boldsymbol{v}}_{2}\right)$ satisfies (3);

(b) $v \in L^{q}\left(\mathbb{R}^{3}\right) \cap D^{1, \frac{2 q}{3}}\left(\mathbb{R}^{3}\right), p \in L^{\frac{3 q}{4}}(\Omega)$, for all $q \in(2, \infty]$;

(c) $v \in X_{e}\left(\mathbb{R}^{3}\right), e:=\tau /|\tau|$;

(d) $v \cdot \nabla v \in \mathcal{V}^{-1}\left(\mathbb{R}^{3}\right)$ and relation (21) is equivalent to the following one:

$$
2[\boldsymbol{v}, \boldsymbol{\varphi}]-\lambda \hat{\boldsymbol{\varphi}}_{1} \cdot \boldsymbol{e}_{1}=\lambda\langle(\tau-\boldsymbol{v}) \cdot \nabla \boldsymbol{v}, \boldsymbol{\varphi}\rangle, \text { for all } \boldsymbol{\varphi} \in \mathcal{V}\left(\mathbb{R}^{3}\right) \text {. }
$$

(e) $v$ obeys the energy equality:

$$
2\|\mathbb{D}(\boldsymbol{v})\|_{2}^{2}=\lambda \tau \cdot e_{1}
$$

Proof. Taking into account that $\Omega$ is of class $C^{\infty}$, the first statement, restricted to $(3)_{1,2,3,5,6}$, follows from the regularity results in [22] (Theorem IX.5.1) applied to (21), along with Remark 2. Next, since $\boldsymbol{\tau} \neq \mathbf{0}$ and $v \in \mathcal{V}\left(\mathbb{R}^{3}\right)$, the results in [22] (Theorem X.6.4) ensure that $v$, and $p$ possess the summability properties stated in (b). In particular, by [22] (Theorem II.9.1), the latter imply also (3) 4 . By Hölder inequality and (16), (17) we show, for all $\varphi \in \mathcal{C}$,

$$
|(\boldsymbol{v} \cdot \nabla \boldsymbol{v}, \varphi)| \leq\|\boldsymbol{v}\|_{3}\|\nabla \boldsymbol{v}\|_{2}\|\varphi\|_{6} \leq \sqrt{2} \kappa_{0}\|\boldsymbol{v}\|_{3}\|\mathbb{D}(\boldsymbol{v})\|_{2}\|\mathbb{D}(\varphi)\|_{2},
$$

which delivers in particular, by (b),

$$
v \cdot \nabla v \in \mathcal{V}^{-1}\left(\mathbb{R}^{3}\right)
$$

Furthermore, from (18), (21) and (26) we infer, for all $\boldsymbol{\varphi} \in \mathcal{C}$,

$$
\begin{aligned}
|(\boldsymbol{\tau} \cdot \nabla \boldsymbol{v}, \boldsymbol{\varphi})| & \leq\|\mathbb{D}(\boldsymbol{v})\|_{2}\|\mathbb{D}(\boldsymbol{\varphi})\|_{2}+\lambda\left[\left|\hat{\boldsymbol{\varphi}}_{1}\right|+\|\boldsymbol{v}\|_{3}\|\nabla \boldsymbol{v}\|_{2}\|\boldsymbol{\varphi}\|_{6}\right] \\
& \leq\left[\|\mathbb{D}(\boldsymbol{v})\|_{2}+\lambda\left(\kappa_{1}+\sqrt{2} \kappa_{0}\|\boldsymbol{v}\|_{3}\|\mathbb{D}(\boldsymbol{v})\|_{2}\right)\right]\|\mathbb{D}(\boldsymbol{\varphi})\|_{2},
\end{aligned}
$$


from which we conclude $v \in X_{e}\left(\mathbb{R}^{3}\right)$. In view of the latter, (27), and the density of $\mathcal{C}$ in $\mathcal{V}\left(\mathbb{R}^{3}\right)$, we may thus deduce that (21) leads to (24). We now replace $v$ for $\boldsymbol{\varphi}$ in (24) to obtain

$$
2\|\mathbb{D}(\boldsymbol{v})\|_{2}^{2}-\lambda \boldsymbol{\tau} \cdot \boldsymbol{e}_{1}=\lambda(\langle\boldsymbol{\tau} \cdot \nabla \boldsymbol{v}, \boldsymbol{v}\rangle-\langle\boldsymbol{v} \cdot \nabla \boldsymbol{v}, \boldsymbol{v}\rangle)=-\lambda\langle\boldsymbol{v} \cdot \nabla \boldsymbol{v}, \boldsymbol{v}\rangle,
$$

where, in the last step, we have taken into account (20). Now, after integrating by parts, we deduce

$$
(v \cdot \nabla v, \varphi)=-(v \cdot \nabla \varphi, v), \text { for all } \varphi \in \mathcal{C} .
$$

Since $v \in L^{4}\left(\mathbb{R}^{3}\right)$ by the Hölder inequality it is readily seen that the right-hand side of (29) defines a continuous functional in $\varphi \in \mathcal{V}(\Omega)$. As a result, setting $\varphi=v$ in (29) we conclude $(v \cdot \nabla v, v)=\langle v \cdot \nabla v, v\rangle=0$, and the property (e) follows from this and (28). The proof of the lemma is completed.

We are now in a position to show the following existence result.

Theorem 2. For any given $\lambda>0$, problem (3) has at least one weak solution $v=v(\lambda)$.

Proof. We shall employ the classical Galerkin method. To this end, let $\left\{\boldsymbol{\varphi}_{k}\right\} \subset \mathcal{C}$ be an ortho-normal basis in $\mathcal{V}\left(\mathbb{R}^{3}\right)$ and, for notational simplicity, set

$$
\widehat{\left(\varphi_{k}\right)_{1}}=\xi_{k}, \quad \widehat{\left(\varphi_{k}\right)_{2}}=\sigma_{k} .
$$

Consider the linear combinations

$$
\boldsymbol{v}_{m}=\sum_{\ell=1}^{m} c_{\ell m} \boldsymbol{\varphi}_{\ell}, \quad \boldsymbol{\tau}_{m}=\sum_{\ell=1}^{m} c_{\ell m} \boldsymbol{\xi}_{\ell}, \boldsymbol{\omega}_{m}=\sum_{\ell=1}^{m} c_{\ell m} \boldsymbol{\sigma}_{\ell}
$$

where the coefficients $c_{\ell m}$ are requested to be solution to the following algebraic set of equations

$$
\left[\boldsymbol{v}_{m}, \boldsymbol{\varphi}_{k}\right]-\lambda \boldsymbol{\xi}_{k} \cdot \boldsymbol{e}_{1}=\lambda\left(\left(\boldsymbol{\tau}_{m}-\boldsymbol{v}_{m}\right) \cdot \nabla \boldsymbol{v}_{m}, \boldsymbol{\varphi}_{k}\right), k \in\{1, \ldots m\} .
$$

Multiplying both sides of this relation by $c_{k m}$ and summing over $k \in\{1, \ldots, m\}$ we show

$$
2\left\|\mathbb{D}\left(\boldsymbol{v}_{m}\right)\right\|_{2}^{2}-\lambda \boldsymbol{\tau}_{m} \cdot \boldsymbol{e}_{1}=\lambda\left(\left(\boldsymbol{\tau}_{m}-\boldsymbol{v}_{m}\right) \cdot \nabla \boldsymbol{v}_{m}, \boldsymbol{v}_{m}\right) .
$$

By a simple integration by parts, we show

$$
\left(\left(\boldsymbol{\tau}_{m}-\boldsymbol{v}_{m}\right) \cdot \nabla \boldsymbol{v}_{m}, \boldsymbol{v}_{m}\right)=0,
$$

and so (31) becomes

$$
2\left\|\mathbb{D}\left(\boldsymbol{v}_{m}\right)\right\|_{2}^{2}-\lambda \boldsymbol{\tau}_{m} \cdot \boldsymbol{e}_{1}=0 .
$$

Now, let $c:=\left(c_{1 m}, \ldots, c_{m m}\right) \in \mathbb{R}^{m}$, and consider the map

$$
\boldsymbol{P}: c \in \mathbb{R}^{m} \mapsto \boldsymbol{P}(\boldsymbol{c}) \in \mathbb{R}^{m},
$$

where

$$
[\boldsymbol{P}(\boldsymbol{c})]_{k}=\left[\boldsymbol{v}_{m}, \boldsymbol{\varphi}_{k}\right]-\lambda \boldsymbol{\xi}_{k} \cdot \boldsymbol{e}_{1}-\lambda\left(\left(\boldsymbol{\tau}_{m}-\boldsymbol{v}_{m}\right) \cdot \nabla \boldsymbol{v}_{m}, \boldsymbol{\varphi}_{k}\right), \quad k \in\{1, \ldots m\} .
$$

By what we have just shown,

$$
\boldsymbol{P}(\boldsymbol{c}) \cdot \boldsymbol{c}=2\left\|\mathbb{D}\left(\boldsymbol{v}_{m}\right)\right\|_{2}^{2}-\lambda \boldsymbol{\tau}_{m} \cdot \boldsymbol{e}_{1}, \text { for all } m \in \mathbb{N},
$$

and so, by (18) and the ortho-normal property of the base $\left\{\boldsymbol{\varphi}_{k}\right\}$,

$$
\boldsymbol{P}(\boldsymbol{c}) \cdot \boldsymbol{c} \geq|\boldsymbol{c}|\left(|\boldsymbol{c}|-\kappa_{1}\right) .
$$


Thus, by [22] (Lemma IX.3.1), the latter implies that for each $m \in \mathbb{N}$, the algebraic system (30) has at least one solution $c=c(m)$. Furthermore, from (32) and again (18) we deduce the following estimate for the sequence $\left\{\boldsymbol{v}_{m}\right\}$, uniformly in $m \in \mathbb{N}$ :

$$
2\left\|\mathbb{D}\left(\boldsymbol{v}_{m}\right)\right\|_{2} \leq \lambda \kappa_{1}
$$

Taking into account that $\mathcal{V}\left(\mathbb{R}^{3}\right) \subset W^{1,2}\left(B_{R}\right)$ and that $W^{1,2}\left(B_{R}\right)$ is compactly embedded in $L^{2}\left(B_{R}\right)$, for all $R>0$, from (33) we deduce the existence of $v \in \mathcal{V}\left(\mathbb{R}^{3}\right)$ such that (possibly along a subsequence)

$$
\begin{aligned}
& \boldsymbol{v}_{m} \rightarrow \boldsymbol{v}, \text { weakly in } \mathcal{V}\left(\mathbb{R}^{3}\right), \text { strongly in } L^{2}\left(B_{R}\right) \text {, all } R>0 \\
& \boldsymbol{\tau}_{m} \rightarrow \boldsymbol{\tau} \equiv \hat{\boldsymbol{v}}_{1}, \boldsymbol{\omega}_{m} \rightarrow \boldsymbol{\omega} \equiv \hat{\boldsymbol{v}}_{2} \text { in } \mathbb{R}^{3} .
\end{aligned}
$$

Employing these convergence properties, we may pass to the limit $m \rightarrow \infty$ in (30) and (32) and show, by classical arguments, that $v$ is a solution to (21) that satisfies, in addition, the "energy inequality:"

$$
2\|\mathbb{D}(v)\|_{2}^{2} \leq \lambda \tau \cdot e_{1} .
$$

From (34) we obtain, in particular that $\boldsymbol{\tau} \neq \mathbf{0}$ because, otherwise, by Lemma $3, \boldsymbol{v} \equiv \mathbf{0}$, in contradiction with (21). Therefore, $v$ is a weak solution to (3) in the sense of Definition 1 , and the proof of the theorem is completed.

Remark 2. A particular subclass of weak solutions to (3) is the one characterized by having $\boldsymbol{\tau}=\tau \boldsymbol{e}_{1}, \tau>0, \boldsymbol{\omega}=\mathbf{0}$, and $\boldsymbol{v}$ possessing rotational symmetry around $\boldsymbol{e}_{1}$. The existence of such solutions is shown in [19] (Theorem 1.1).

The previous theorem proves the existence of a steady-state solution, $v=v(\lambda)$, to (1) for all $\lambda>0$. However, it is silent about the regularity of the map $\lambda \mapsto v(\lambda)$. In the next theorem, we shall furnish sufficient conditions for the existence of a local, unique, analytic family of weak solutions for which the velocity of the center of mass is directed along the same direction. To this end, let $v_{c}$ be a weak solution to (3) corresponding to $\lambda=\lambda_{\mathrm{c}}$, denote by $\tau_{\mathrm{c}}=\tau_{\mathrm{c}} \boldsymbol{e}_{\mathrm{c}}$ the corresponding translational velocity, where $\boldsymbol{e}_{\mathrm{c}}:=\tau_{\mathrm{c}} /\left|\boldsymbol{\tau}_{\mathrm{c}}\right|$. Moreover, define

$$
\mathcal{V}_{\mathrm{c}}=\mathcal{V}_{\mathrm{c}}\left(\mathbb{R}^{3}\right):=\left\{\boldsymbol{u} \in \mathcal{V}\left(\mathbb{R}^{3}\right): \boldsymbol{u}(x)=\hat{u}_{1} \boldsymbol{e}_{\mathrm{c}}+\hat{\boldsymbol{u}}_{2} \times x, x \in \Omega_{0}\right\},
$$

and set

$$
x_{\mathrm{c}}\left(\mathbb{R}^{3}\right)=\mathcal{V}_{\mathrm{c}}\left(\mathbb{R}^{3}\right) \cap \chi_{\boldsymbol{e}_{\mathrm{c}}}\left(\mathbb{R}^{3}\right) .
$$

We can then prove that the map $\lambda \mapsto v(\lambda)$ is smooth in a neighborhood of $\lambda_{\mathrm{c}}$ in the class $x_{c}$, provided the linearization of (21) around $\left(v_{c}, \lambda_{c}\right)$ is trivial. To this end, let

$$
\mathcal{L}_{1}: u \in X_{\mathrm{c}}\left(\mathbb{R}^{3}\right) \mapsto \mathcal{L}_{1}(u) \in \mathcal{V}^{-1}\left(\mathbb{R}^{3}\right)
$$

where

$$
\begin{gathered}
\left\langle\mathcal{L}_{1}(\boldsymbol{u}), \boldsymbol{\varphi}\right\rangle=2[\boldsymbol{u}, \boldsymbol{\varphi}]-\lambda_{\mathrm{c}}\left[\tau_{\mathrm{c}}\left\langle\boldsymbol{e}_{\mathrm{c}} \cdot \nabla \boldsymbol{u}, \boldsymbol{\varphi}\right\rangle+\hat{u}_{1}\left\langle\boldsymbol{e}_{\mathrm{c}} \cdot \nabla \boldsymbol{v}_{\mathrm{c}}, \boldsymbol{\varphi}\right\rangle\right. \\
\left.+\left(\boldsymbol{u} \cdot \nabla \boldsymbol{v}_{\mathrm{c}}+\boldsymbol{v}_{\mathrm{c}} \cdot \nabla \boldsymbol{u}, \boldsymbol{\varphi}\right)\right], \boldsymbol{\varphi} \in \mathcal{V}\left(\mathbb{R}^{3}\right) .
\end{gathered}
$$

The following result holds.

Theorem 3. Let $\boldsymbol{v}_{\mathrm{c}}$ be a weak solution to (3) corresponding to $\lambda=\lambda_{\mathrm{c}}$. Then, the operator $\mathcal{L}_{1}$ is Fredholm of index 0. Furthermore, suppose that the equation

$$
\mathcal{L}_{1}(u)=0
$$

has only the solution $\boldsymbol{u}=\mathbf{0}$ in $X_{\mathrm{c}}\left(\mathbb{R}^{3}\right)$. Then, there exists a neighborhood $U_{\mathrm{c}}$ of $\lambda_{\mathrm{c}}$, such that (3) has a unique family of weak solutions $v(\lambda) \in X_{c}\left(\mathbb{R}^{3}\right), \lambda \in U_{c}$, which is analytic at $\lambda_{c}$ and such that $v\left(\lambda_{\mathrm{c}}\right)=\boldsymbol{v}_{\mathrm{c}}$. 
Proof. Consider the map

$$
\mathcal{F}:(v, \lambda) \in X_{\mathrm{c}} \times U_{\mathrm{c}} \mapsto \mathcal{F}(\boldsymbol{v}, \lambda) \in \mathcal{V}^{-1},
$$

where

$$
\langle\mathcal{F}(v, \lambda), \varphi\rangle:=[v, \varphi]-\lambda \hat{\varphi}_{1} \cdot e_{1}-\lambda\left\langle\left(\hat{v}_{1} e_{\mathrm{c}}-v\right) \cdot \nabla v, \varphi\right\rangle, \varphi \in \mathcal{V} .
$$

The map is well defined. In fact, since $v \in X_{c}$, we have $e_{c} \cdot \nabla v \in \mathcal{V}^{-1}$. Furthermore, from (29), Hölder inequality and Lemma 4 it follows that $v \cdot \nabla v \in \mathcal{V}^{-1}$ as well. In addition, since $\mathcal{F}$ involves only cubic nonlinearities, $\mathcal{F}$ is analytic. We now observe that (24) is equivalent to $\mathcal{F}(v, \lambda)=0$. In [19] (Lemmas 2.2 and 2.3) it is shown that the operator

$$
\mathcal{O}: \boldsymbol{u} \in \mathcal{X}_{\mathrm{c}} \mapsto \mathcal{O}(\boldsymbol{u}) \in \mathcal{V}^{-1},
$$

with

$$
\langle\mathcal{O}(u), \varphi\rangle:=[u, \varphi]-\lambda_{c}\left\langle\tau_{c} e_{c} \cdot \nabla u, \varphi\right\rangle, \varphi \in \mathcal{V},
$$

is a homeomorphism, while the operator

$$
\mathcal{K}: \boldsymbol{u} \in \mathcal{X}_{\mathrm{c}} \mapsto \mathcal{K}(\boldsymbol{u}) \in \mathcal{V}^{-1},
$$

with

$$
\langle\mathcal{K}(\boldsymbol{u}), \boldsymbol{\varphi}\rangle:=-\lambda_{\mathrm{c}}\left\langle\left(\hat{u}_{1} e_{\mathrm{c}}-\boldsymbol{u}\right) \cdot \nabla \boldsymbol{v}_{\mathrm{c}}-\boldsymbol{v}_{\mathrm{c}} \cdot \nabla \boldsymbol{u}, \boldsymbol{\varphi}\right\rangle, \boldsymbol{\varphi} \in \mathcal{V},
$$

is compact. As a consequence, $\mathcal{L}_{1} \equiv \mathcal{O}+\mathcal{K}$ is Fredholm of index 0 and thus, under the assumptions of the theorem, it is a homeomorphism. By Lemma $5(\mathrm{~d})$, it is $\mathcal{F}\left(\boldsymbol{v}_{\mathrm{c}}, \lambda_{\mathrm{c}}\right)=0$, while the partial Fréchet derivative of $\mathcal{F}$ at $\left(v_{c}, \lambda_{c}\right), D_{v} \mathcal{F}\left(v_{c}, \lambda_{c}\right)$, is easily shown to satisfy $D_{v} \mathcal{F}\left(v_{c}, \lambda_{c}\right)=\mathcal{L}_{1}$. Since $\mathcal{L}_{1}$ is a homeomorphism, the result is an immediate consequence of the analytic version of the Implicit Function Theorem.

Remark 3. We observe that the assumption of Theorem 3 excludes that $\left(v_{\mathrm{c}}, \lambda_{\mathrm{c}}\right)$ is a steady-state bifurcation point in the class $X_{c}$ [19].

\section{On the Spectral Properties of the Linearized Operator}

The main objective of this section is to establish some important spectral properties of the operator obtained by linearizing (3) around the solution $v_{c}$. As shown later on, such properties will support one of the basic assumptions of our bifurcation result. To this end, we begin to define the map

$$
\widetilde{\Delta}: \boldsymbol{w} \in W^{2,2}(\Omega) \cap \mathcal{L}^{2}\left(\mathbb{R}^{3}\right) \mapsto \widetilde{\Delta}(\boldsymbol{w}) \in \mathcal{L}^{2}\left(\mathbb{R}^{3}\right)
$$

where

$$
\widetilde{\Delta}(\boldsymbol{w})=\left\{\begin{array}{l}
-\Delta w \text { in } \Omega, \\
\frac{1}{M} \int_{\partial \Omega}(2 \mathbb{D}(\boldsymbol{w}) \cdot \boldsymbol{n})+\left(\frac{1}{\mathcal{I}} \int_{\partial \Omega} y \times(2 \mathbb{D}(w) \cdot n)\right) \times x \text { in } \Omega_{0},
\end{array}\right.
$$

and set $\mathcal{A}:=\mathcal{P} \widetilde{\Delta}$, with $\mathcal{P}$ the orthogonal projection of $\mathcal{L}^{2}\left(\mathbb{R}^{3}\right)$ onto $\mathcal{H}\left(\mathbb{R}^{3}\right)$; see Lemma 2 . We next consider the operator

$$
\mathcal{L}_{0}: \boldsymbol{u} \in Z^{2,2}:=W^{2.2}(\Omega) \cap \mathcal{H}\left(\mathbb{R}^{3}\right) \mapsto \mathcal{L}_{0}(\boldsymbol{u})=-\lambda_{\mathrm{c}} \boldsymbol{\tau}_{\mathrm{c}} \cdot \nabla \boldsymbol{u}+\mathcal{A}(\boldsymbol{u}) \in \mathcal{H}\left(\mathbb{R}^{3}\right) .
$$

$\mathcal{L}_{0}$ is well defined since

$$
\boldsymbol{\tau}_{\mathrm{c}} \cdot \nabla \boldsymbol{u} \in \mathcal{H}\left(\mathbb{R}^{3}\right) .
$$

In fact, we observe that $u \in Z^{2,2}$ implies $u \in \mathcal{V}\left(\mathbb{R}^{3}\right)$, so that by Lemma 2 and (12), it follows that (41) reduces to prove that 


$$
\int_{\Omega} \boldsymbol{\tau}_{\mathrm{c}} \cdot \nabla \boldsymbol{u} \cdot \boldsymbol{h}+M\left(\widehat{\boldsymbol{\tau}_{\mathrm{c}} \cdot \nabla \boldsymbol{u}}\right)_{1} \cdot \hat{\boldsymbol{h}}_{1}+\mathcal{I}\left(\widehat{\boldsymbol{\tau}_{\mathrm{c}} \cdot \nabla \boldsymbol{u}}\right)_{2} \cdot \hat{\boldsymbol{h}}_{2}=0, \text { for all } \boldsymbol{h} \in \mathcal{G}\left(\mathbb{R}^{3}\right) .
$$

Taking into account that, in $\Omega_{0}$, it is $\tau_{\mathrm{c}} \cdot \nabla \boldsymbol{u}=\boldsymbol{\tau}_{\mathrm{c}} \cdot \nabla\left(\hat{\boldsymbol{u}}_{2} \times \boldsymbol{x}\right)=\hat{\boldsymbol{u}}_{2} \times \boldsymbol{\tau}_{\mathrm{c}}$, while, by $(13)_{3}, \boldsymbol{h}=\nabla p$ in $\Omega$ with $\hat{\boldsymbol{h}}_{1}=-M^{-1} \int_{\partial \Omega} p \boldsymbol{n}$ and $\hat{\boldsymbol{h}}_{2}=-\mathcal{I}^{-1} \int_{\partial \Omega} p \boldsymbol{x} \times \boldsymbol{n}$, (42) becomes

$$
\int_{\Omega} \boldsymbol{\tau}_{\mathrm{c}} \cdot \nabla \boldsymbol{u} \cdot \nabla p-\int_{\partial \Omega} p \hat{\boldsymbol{u}}_{2} \times \boldsymbol{\tau}_{\mathrm{c}} \cdot \boldsymbol{n}=0,
$$

whose validity is immediately checked by integrating by parts the volume integral, and recalling that $\operatorname{div} \boldsymbol{u}=0$.

The following preliminary result holds.

Lemma 6. Let $\zeta \in \mathbb{R} \backslash\{0\}$ and $\left(\lambda_{c}, \boldsymbol{\tau}_{\mathrm{c}}\right) \in \mathbb{R} \times \mathbb{R}^{3}$. Then, the operator $\mathcal{L}_{0}+\beta \zeta$ is a homeomorphism of $Z_{\mathbb{C}}^{2,2}$ onto $\mathcal{H}_{\mathbb{C}}\left(\mathbb{R}^{3}\right)$. Moreover, there is $c=c\left(\Omega, \rho_{\mathcal{S}} / \rho_{\mathcal{L}}\right)$, such that

$$
\begin{gathered}
\left\|D^{2} \boldsymbol{u}\right\|_{2}+|\zeta|^{\frac{1}{2}}\|\nabla \boldsymbol{u}\|_{2}+|\zeta|\left(\|\boldsymbol{u}\|_{2}+M^{\frac{1}{2}}|\chi|+\mathcal{I}^{\frac{1}{2}}|\sigma|\right) \leq c\left\|\left(\mathcal{L}_{0}+B \zeta\right)(\boldsymbol{u})\right\|_{2}, \\
|\zeta| \geq \max \left\{\lambda_{c}^{2}\left|\boldsymbol{\tau}_{\mathrm{c}}\right|^{2}, 1\right\},
\end{gathered}
$$

where $\chi:=\hat{\boldsymbol{u}}_{1}, \sigma:=\hat{\boldsymbol{u}}_{2}$.

Proof. The homeomorphism property can be obtained by proving that, for any $\mathcal{F} \in$ $\mathcal{H}_{\mathbb{C}}\left(\mathbb{R}^{3}\right)$, there exists one and only one $\boldsymbol{u} \in Z_{\mathbb{C}}^{2,2}$ such that $\mathcal{L}_{0}(\boldsymbol{u})+\beta \zeta \boldsymbol{u}=\mathcal{F}$. In view of Lemma 1, Lemma 2 and (39) this is equivalent to requiring that for any $(f, F, G) \in$ $L_{\mathbb{C}}^{2}(\Omega) \times \mathbb{C} \times \mathbb{C}$, with $\operatorname{div} f=0$, the problem

$$
\begin{gathered}
\left.\begin{array}{c}
\Delta \boldsymbol{u}+\lambda_{\mathrm{c}} \boldsymbol{\tau}_{\mathrm{c}} \cdot \nabla \boldsymbol{u}-\nabla \mathfrak{p}=\S \zeta \boldsymbol{u}+\boldsymbol{f} \\
\operatorname{div} \boldsymbol{u}=0
\end{array}\right\} \operatorname{in} \Omega, \\
\boldsymbol{u}=\chi+\boldsymbol{\sigma} \times \boldsymbol{x} \text { at } \partial \Omega, \\
\S \zeta M \chi+\int_{\partial \Omega} \mathbb{T}(\boldsymbol{u}, \mathfrak{p}) \cdot \boldsymbol{n}=\boldsymbol{F}, \S \zeta \mathcal{I} \boldsymbol{\sigma}+\int_{\partial \Omega} \boldsymbol{x} \times \mathbb{T}(\boldsymbol{u}, \mathfrak{p}) \cdot \boldsymbol{n}=\boldsymbol{G},
\end{gathered}
$$

has one and only one solution $(\boldsymbol{u}, \mathfrak{p}, \boldsymbol{\chi}, \boldsymbol{\sigma}) \in W_{\mathbb{C}}^{2,2}(\Omega) \times D_{\mathbb{C}}^{1,2}(\Omega) \times \mathbb{C} \times \mathbb{C}$. Let us dotmultiply both sides of $(44)_{1}$ by $\boldsymbol{u}^{*}\left({ }^{*}=\right.$ complex conjugate) and integrate by parts over $\Omega$. Taking into account $(44)_{2-5}$ we show

$$
2\|\mathbb{D}(\boldsymbol{u})\|_{2}^{2}+B \zeta\left(\|\boldsymbol{u}\|_{2}^{2}+M|\chi|^{2}+\mathcal{I}|\sigma|^{2}\right)-\lambda\left(\boldsymbol{\tau} \cdot \nabla \boldsymbol{u}, \boldsymbol{u}^{*}\right)=\left(\boldsymbol{f}, \boldsymbol{u}^{*}\right)+\boldsymbol{F} \cdot \boldsymbol{\chi}^{*}+\boldsymbol{G} \cdot \boldsymbol{\sigma}^{*},
$$

where, here and in what follows, in order to simplify the notation we suppress the subscript c. Taking the real and imaginary parts of (43), using (16) and Schwarz inequality, and observing that $\Re\left(\boldsymbol{\tau} \cdot \nabla \boldsymbol{u}, \boldsymbol{u}^{*}\right)=0$, we deduce

$$
\|\nabla \boldsymbol{u}\|_{2}^{2} \leq \mathcal{F} \boldsymbol{W} ; \quad|\zeta|\|\boldsymbol{u}\|^{2} \leq\left(\lambda|\boldsymbol{\tau}|\|\nabla \boldsymbol{u}\|_{2}+\mathcal{F}\right) \boldsymbol{W},
$$

where

$$
\begin{aligned}
& \|\boldsymbol{W}\|:=\left(\|\boldsymbol{u}\|_{2}^{2}+M|\chi|^{2}+\mathcal{I}|\sigma|^{2}\right)^{\frac{1}{2}} \\
& \mathcal{W}:=\|\boldsymbol{u}\|_{2}+M^{\frac{1}{2}}|\chi|+\mathcal{I}^{\frac{1}{2}}|\boldsymbol{\sigma}| \\
& \mathcal{F}:=\|\boldsymbol{f}\|_{2}+M^{-\frac{1}{2}}|\boldsymbol{F}|+\mathcal{I}^{-\frac{1}{2}}|\boldsymbol{G}| .
\end{aligned}
$$

Observing that $\boldsymbol{W} \leq 3^{\frac{1}{2}}\|\boldsymbol{W}\|$, from (46) and Cauchy-Schwarz inequality we get

$$
|\zeta|\|\boldsymbol{u}\| \leq 3^{\frac{1}{2}}\left(\lambda|\boldsymbol{\tau}| \mathcal{F}^{\frac{1}{2}} \boldsymbol{W}^{\frac{1}{2}}+\mathcal{F}\right) \leq 3^{\frac{1}{2}}\left(\frac{3 \lambda^{2}|\boldsymbol{\tau}|^{2}}{|\zeta|}+1\right) \mathcal{F}+\frac{1}{2}|\zeta|\|\boldsymbol{W}\|,
$$


from which we conclude

$$
|\zeta|\|\boldsymbol{W}\| \leq 3^{\frac{1}{2}}\left(\frac{3 \lambda^{2}|\boldsymbol{\tau}|^{2}}{|\zeta|}+2\right) \mathcal{F} .
$$

Replacing the latter into (46) 1 , we infer

$$
|\zeta|^{\frac{1}{2}}\|\nabla \boldsymbol{u}\|_{2} \leq 3^{\frac{1}{4}}\left(\frac{3 \lambda^{2}|\boldsymbol{\tau}|^{2}}{|\zeta|}+2\right)^{\frac{1}{2}} \mathcal{F} .
$$

Combining the estimates (47) and (48) with classical Galerkin method, we can proceed as in the proof of Theorem 2 and show that for any given $(f, F, G)$ in the specified class and $\zeta \neq 0$, there exists a (unique, weak) solution to (44) such that $u \in \mathcal{V}_{\mathbb{C}}(\Omega) \cap L_{\mathbb{C}}^{2}(\Omega)$, satisfying (47) and (48). We next write (44) $1_{-3}$ as the following Stokes problem

$$
\begin{aligned}
& \begin{array}{l}
\Delta \boldsymbol{u}=\nabla \mathfrak{p}+\mathcal{G} \\
\operatorname{div} \boldsymbol{u}=0
\end{array} \\
& \boldsymbol{u}=\boldsymbol{\chi}+\boldsymbol{\sigma} \times \boldsymbol{x} \text { at } \partial \Omega
\end{aligned} \text { in }
$$

where

$$
\mathcal{G}:=-\lambda \boldsymbol{\tau} \cdot \nabla \boldsymbol{u}+\beta \zeta \boldsymbol{u}+f .
$$

Since $\mathcal{G} \in L_{\mathbb{C}}^{2}(\Omega)$ and $\boldsymbol{u} \in W_{\mathbb{C}}^{1,2}(\Omega)$, from classical results [22] (Theorems IV.5.1 and V.5.3) it follows that $D^{2} \boldsymbol{u} \in L^{2}(\Omega)$, thus completing the existence (and uniqueness) proof. Furthermore, by [22] (Lemma IV.1.1 and V.4.3) we get

$$
\left\|D^{2} \boldsymbol{u}\right\|_{2} \leq c\left[\|\boldsymbol{f}\|_{2}+(\lambda|\boldsymbol{\tau}|+1)\|\nabla \boldsymbol{u}\|_{2}+(|\zeta|+1)\|\boldsymbol{u}\|_{2}+|\chi|+|\boldsymbol{\sigma}|\right] .
$$

As a result, if $|\zeta| \geq \max \left\{\lambda^{2}|\tau|^{2}, 1\right\}$, the inequality in (43) is a consequence of (47)-(49).

Let

$$
\mathcal{K}: u \in Z^{2,2} \mapsto \mathcal{K}(u) \in \mathcal{L}^{2}\left(\mathbb{R}^{3}\right)
$$

where $\left(\chi:=\hat{\boldsymbol{u}}_{1}\right)$,

$$
\mathcal{K}(\boldsymbol{u})=\left\{\begin{array}{l}
\lambda_{\mathrm{c}}\left(\boldsymbol{v}_{\mathrm{c}} \cdot \nabla \boldsymbol{u}+(\boldsymbol{u}-\chi) \cdot \nabla \boldsymbol{v}_{\mathrm{c}}\right) \text { in } \Omega, \\
\mathbf{0} \text { in } \Omega_{0} .
\end{array}\right.
$$

From Lemma 5, we get, in particular,

$$
v_{\mathrm{c}} \in L^{\infty}(\Omega) \cap L^{4}(\Omega), \quad \nabla v_{\mathrm{c}} \in L^{\infty}(\Omega) \cap D^{1,2}(\Omega),
$$

and so we easily check that the operator $\mathcal{K}$ is well defined. Finally, let

$$
\mathcal{L}_{2}: \boldsymbol{u} \in W^{2,2}(\Omega) \cap \mathcal{H}\left(\mathbb{R}^{3}\right) \mapsto \mathcal{L}_{2}(\boldsymbol{u})=-\lambda_{\mathrm{c}} \boldsymbol{\tau}_{\mathrm{c}} \cdot \nabla \boldsymbol{u}+\mathcal{A}(\boldsymbol{u})+\mathcal{P} \mathcal{K}(\boldsymbol{u}) \in \mathcal{H}\left(\mathbb{R}^{3}\right) .
$$

We are now ready to show the main result of this section.

Theorem 4. The operator $\mathcal{L}_{2}+B \zeta$ is Fredholm of index 0 , for all $\zeta \neq 0$. Moreover, let $\sigma\left(\mathcal{L}_{2}\right)$ be the spectrum of $\mathcal{L}_{2}$. Then, $\sigma\left(\mathcal{L}_{2}\right) \cap\{B \mathbb{R} \backslash\{0\}\}$ consists, at most, of a finite or countable number of eigenvalues, each of which is isolated and of finite (algebraic) multiplicity, that can only accumulate at 0 .

Proof. We show that the operator $\mathcal{K}$ defined in (50), (51) is compact. Let $\left\{\boldsymbol{u}_{k}\right\}$ be a sequence bounded in $Z^{2,2}$. This implies, in particular, that there is $M>0$ independent of $k$ such that $\left(\chi_{k}:=\widehat{\left(\boldsymbol{u}_{k}\right)_{1}}\right)$

$$
\left\|\boldsymbol{u}_{k}\right\|_{2,2}+\left|\chi_{k}\right| \leq M \text {. }
$$


The latter, along with the compact embedding $W^{2,2}(\Omega) \subset W^{1,2}\left(\Omega_{R}\right)$, for all $R>1$ entails the existence of $\left(\boldsymbol{u}_{*}, \boldsymbol{\chi}_{*}\right) \in W^{2,2}(\Omega) \times \mathbb{R}$ and subsequences, again denoted by $\left\{\boldsymbol{u}_{k}, \boldsymbol{\chi}_{k}\right)$ such that

$$
\boldsymbol{u}_{k} \rightarrow \boldsymbol{u}_{*} \text { strongly in } W^{1,2}\left(\Omega_{R}\right) \text {, for all } R>1 ; \chi_{k} \rightarrow \chi_{*} \text { in } \mathbb{R}^{3} .
$$

Without loss, we assume $\boldsymbol{u}_{*} \equiv \chi_{*} \equiv \mathbf{0}$. By Hölder inequality and (50), we deduce

$$
\left\|\mathcal{K}\left(\boldsymbol{u}_{k}\right)\right\|_{2} \leq \lambda_{\mathrm{c}}\left[\left(\left\|\boldsymbol{v}_{\mathrm{c}}\right\|_{\infty}+\left\|\nabla \boldsymbol{v}_{\mathrm{c}}\right\|_{\infty}\right)\left(\left\|\boldsymbol{u}_{k}\right\|_{1,2, \Omega_{R}}+\left|\chi_{k}\right|\right)+\left\|\boldsymbol{v}_{\mathrm{c}}\right\|_{1,4, \Omega^{R}}\left\|\nabla \boldsymbol{u}_{k}\right\|_{1,4}\right] .
$$

Therefore, passing to the limit $k \rightarrow \infty$ in the previous inequality, and using (52), (54) and (55) and the embedding $W^{2,2} \subset W^{1,4}$, we infer

$$
\lim _{k \rightarrow \infty}\left\|\mathcal{K}\left(\boldsymbol{u}_{k}\right)\right\|_{2} \leq M\left\|\boldsymbol{v}_{\mathrm{c}}\right\|_{1,4, \Omega^{R}}
$$

which, in turn, again by (52) and the arbitrariness of $R>1$ shows the desired property. From this and Lemma 6 it then follows that the operator

$$
\widehat{\mathcal{L}}_{\zeta}:=\mathcal{L}_{1}+\beta \zeta
$$

is Fredholm of index 0 , for all $\zeta \neq 0$. The theorem is then a consequence of well-known results (e.g., [23] (Theorem XVII.4.3)) provided we show that the null space of $\widehat{\mathcal{L}}_{\zeta}$ is trivial, for all sufficiently large $|\zeta|$. To this end, we observe that $\widehat{\mathcal{L}}_{\zeta}(\boldsymbol{u})=0$ means $\mathcal{L}_{0}(\boldsymbol{u})+\beta \zeta \boldsymbol{u}=$ $-\mathcal{K}(\boldsymbol{u})$. Applying (43), we thus get, in particular, the following inequality valid for all sufficiently large $|\zeta|$

$$
|\zeta|^{\frac{1}{2}}\|\nabla \boldsymbol{u}\|_{2}+|\zeta|\left(\|\boldsymbol{u}\|_{2}+|\chi|\right) \leq c\|\mathcal{K}(\boldsymbol{u})\|_{2},
$$

where $c$ is independent of $\zeta$. Also, from (50)-(52) and Hölder inequality, we have

$$
\|\mathcal{K}(\boldsymbol{u})\|_{2} \leq \lambda_{\mathrm{c}}\left\|\boldsymbol{v}_{\mathrm{c}}\right\|_{1, \infty}\left(\|\boldsymbol{u}\|_{1,2}+|\chi|\right),
$$

and so, from the last two displayed equations we deduce $\boldsymbol{u} \equiv \mathbf{0}$, provided we choose $|\zeta|$ larger than a suitable positive constant depending only on $\Omega, \lambda_{c}, M$, and $\mathcal{I}$. This completes the proof of the theorem.

\section{On the Linearized Time-Periodic Operator}

Let

$$
\begin{aligned}
& \mathfrak{L}_{\sharp}^{2}:=\left\{\mathcal{F} \in L^{2}\left(\mathcal{L}^{2}\right), \mathcal{F} \text { is } 2 \pi \text {-periodic with } \overline{\left.\mathcal{F}\right|_{\Omega}}=\overline{\widehat{\mathcal{F}}_{1}}=\overline{\widehat{\mathcal{F}}_{2}}=\mathbf{0}\right\}, \\
& \mathfrak{H}_{\sharp}:=\left\{f \in \mathfrak{L}_{\sharp}^{2}: f \in L^{2}(\mathcal{H})\right\} \\
& \mathfrak{W}_{\sharp}^{2}:=\left\{\boldsymbol{w} \in \mathcal{W}_{\sharp}^{2}: \boldsymbol{u} \in W^{1,2}(\mathcal{H})\right\},
\end{aligned}
$$

and, for $\zeta_{0} \neq 0$, consider the operators

$$
\mathcal{Q}_{0}: w \in \mathfrak{W}_{\sharp}^{2} \mapsto \zeta_{0} \partial_{\mathrm{s}} \boldsymbol{w}+\mathcal{L}_{0}(\boldsymbol{w}) \in \mathfrak{H}_{\sharp},
$$

and

$$
\mathcal{Q}: w \in \mathfrak{W}_{\sharp}^{2} \mapsto \zeta_{0} \partial_{\mathrm{s}} w+\mathcal{L}_{2}(w) \in \mathfrak{H}_{\sharp},
$$

where $\mathcal{L}_{0}$ and $\mathcal{L}_{2}$ are given in (40) and (53), respectively.

Our objective in this section is to establish some important properties for both operators. We begin to show the following lemma. 
Lemma 7. Let $\tau \in \mathbb{R}$. Then, the boundary-value problems, $i \in\{1,2,3\}, k \in \mathbb{Z} \backslash\{0\}$,

$$
\begin{aligned}
& \mathrm{i} k \boldsymbol{h}_{k}^{(i)}-\tau \partial_{1} \boldsymbol{h}_{k}^{(i)}=\Delta \boldsymbol{h}_{k}^{(i)}-\nabla p_{k}^{(i)} \\
& \operatorname{div} \boldsymbol{h}_{k}^{(i)}=0 \\
& \multicolumn{2}{c}{\boldsymbol{h}_{k}^{(i)}=\boldsymbol{e}_{i} \text { at } \partial \Omega, \boldsymbol{h}_{0}^{(i)}=\mathbf{0}}
\end{aligned}
$$

and

$$
\begin{aligned}
& \begin{array}{l}
\operatorname{i} k \boldsymbol{H}_{k}^{(i)}-\tau \partial_{1} \boldsymbol{H}_{k}^{(i)}=\Delta \boldsymbol{H}_{k}^{(i)}-\nabla P_{k}^{(i)} \\
\operatorname{div} \boldsymbol{H}_{k}^{(i)}=0 \\
\quad \boldsymbol{H}_{k}^{(i)}=\boldsymbol{e}_{i} \times \boldsymbol{x} \text { at } \partial \Omega, \boldsymbol{H}_{0}^{(i)}=\mathbf{0}
\end{array} \text { in } \Omega
\end{aligned}
$$

have unique solutions $\left(\boldsymbol{h}_{k}^{(i)}, p_{k}^{(i)}\right),\left(\boldsymbol{H}_{k}^{(i)}, P_{k}^{(i)}\right) \in W^{2,2}(\Omega) \times D^{1,2}(\Omega)$. These solutions satisfy the estimates

$$
\begin{aligned}
& \left\|\boldsymbol{h}_{k}^{(i)}\right\|_{2}+\left\|\boldsymbol{H}_{k}^{(i)}\right\|_{2} \leq C \\
& \left\|\nabla \boldsymbol{h}_{k}^{(i)}\right\|_{2}+\left\|\nabla \boldsymbol{H}_{k}^{(i)}\right\|_{2} \leq C(|k|+1)^{\frac{1}{2}} \\
& \left\|D^{2} \boldsymbol{h}_{k}^{(i)}\right\|_{2}+\left\|D^{2} \boldsymbol{H}_{k}^{(i)}\right\|_{2} \leq C(|k|+1),
\end{aligned}
$$

where $C$ is a constant independent of $k$. Moreover, for fixed $k$, consider the $3 \times 3$ matrices $\mathbb{K}, \mathbb{A}, \mathbb{P}$ and $\mathbb{S}$ defined by the components $(j, i=1,2,3)$ :

$$
\begin{gathered}
(\mathbb{K})_{j i}=\int_{\Sigma}\left(\mathbb{T}\left(\boldsymbol{h}_{k}^{(i)}, p_{k}^{(i)}\right) \cdot \boldsymbol{n}\right)_{j}, \\
(\mathbb{A})_{j i}=\int_{\Sigma}\left(\boldsymbol{x} \times \mathbb{T}\left(\boldsymbol{H}_{k}^{(i)}, P_{k}^{(i)}\right) \cdot \boldsymbol{n}\right)_{j} \\
(\mathbb{P})_{j i}=\int_{\Sigma}\left(\boldsymbol{x} \times \mathbb{T}\left(\boldsymbol{h}_{k}^{(i)}, p_{k}^{(i)}\right) \cdot \boldsymbol{n}\right)_{j} \\
(\mathbb{S})_{j i}=\int_{\Sigma}\left(\mathbb{T}\left(\boldsymbol{H}_{k}^{(i)}, P_{k}^{(i)}\right) \cdot \boldsymbol{n}\right)_{j}
\end{gathered}
$$

and define the $6 \times 6$ matrix $\mathcal{A}$ as follows

$$
\mathcal{A}:=\left(\begin{array}{cc}
\mathbb{K} & \mathbb{P} \\
\mathbb{S} & \mathbb{A}
\end{array}\right)
$$
we have

Then, for any $\mu \in \mathbb{R}$, both $\mathbb{K}+ß \mu \mathbb{1}$ and $\mathbb{A}+\beta \mu \mathbb{1}$ are invertible. Moreover, for every $\zeta \in \mathbb{C}^{6}$,

$$
\mathrm{i} k\|\boldsymbol{v}\|_{2}^{2}+2\|\mathbb{D}(\boldsymbol{v})\|_{2}^{2}-\tau\left(\partial_{1} \boldsymbol{v}, \boldsymbol{v}^{*}\right)=\boldsymbol{\zeta}^{*} \cdot \boldsymbol{A} \cdot \boldsymbol{\zeta}
$$

where $\boldsymbol{v}:=\zeta_{i} \boldsymbol{h}_{k}^{(i)}+\zeta_{i+3} \boldsymbol{H}_{k}^{(i)}$. Finally, for every $(\lambda, \mu) \in \mathbb{R} \times \mathbb{R}$, the matrix

$$
\mathcal{A}+\left(\begin{array}{cc}
B \lambda \mathbb{I} & 0 \\
0 & B \mu \mathbb{I}
\end{array}\right):=\boldsymbol{A}+\boldsymbol{g}
$$

is invertible.

Proof. We begin to show the estimate for $\boldsymbol{h}_{k}^{(i)}$. Since the proof is the same for $i=1,2,3$, we chose $i=1$ and, for simplicity, omit the superscript. Let $\phi=\phi(|x|)$ be a (smooth) cut-off function such that

$$
\phi(|x|)=\left\{\begin{array}{l}
1 \text { in } \Omega_{R} \\
0 \text { in } \overline{\Omega^{2 R}}
\end{array}\right.
$$


and set $\boldsymbol{\Phi}(x)=\operatorname{curl}\left(x_{2} \phi(|x|) \boldsymbol{e}_{3}\right)$. Clearly, $\operatorname{div} \boldsymbol{\Phi}=0$ and $\boldsymbol{\Phi}(x)=\boldsymbol{e}_{1}$ in a neighborhood of $\partial \Omega$. Moreover $\boldsymbol{\Phi}(x) \equiv \mathbf{0}$ in $\Omega^{2 R}$. Setting $\boldsymbol{v}_{k}:=\boldsymbol{h}_{k}-\boldsymbol{\Phi}$, from (57) we deduce that $\boldsymbol{v}_{k}$ solves the following boundary-value problem, for all $|k| \geq 1$ :

$$
\left.\begin{array}{c}
\begin{array}{l}
\mathrm{i} k \boldsymbol{v}_{k}-\tau \partial_{1} \boldsymbol{v}_{k}=\Delta \boldsymbol{v}_{k}-\nabla p_{k}^{(i)}+\tau \partial_{1} \boldsymbol{\Phi}-\mathrm{i} k \boldsymbol{\Phi}+\Delta \boldsymbol{\Phi} \\
\operatorname{div} \boldsymbol{v}_{k}=0
\end{array} \\
\boldsymbol{v}_{k}=\mathbf{0} \text { at } \partial \Omega .
\end{array}\right\} \text { in } \Omega
$$

Existence of (62) in the stated function class can be easily obtained by the Galerkin method combined with the estimate that we are about to derive. Let us dot-multiply both sides of $(62)_{1}$ by $v^{*}$ where the star denotes c.c. After integrating by parts as necessary, we get

$$
\mathrm{i} k\left\|\boldsymbol{v}_{k}\right\|_{2}^{2}-\tau\left(\partial_{1} \boldsymbol{v}, \boldsymbol{v}_{k}^{*}\right)+\left\|\nabla \boldsymbol{v}_{k}\right\|_{2}^{2}=\left(\mathcal{F}_{k}, \boldsymbol{v}_{k}^{*}\right),
$$

where $\mathcal{F}_{k}:=\tau \partial_{1} \boldsymbol{\Phi}-\mathrm{i} k \boldsymbol{\Phi}+\Delta \boldsymbol{\Phi}$. We next observe that, by the properties of $\boldsymbol{\Phi}$,

$$
\left\|\mathcal{F}_{k}\right\|_{2} \leq c(|k|+1)
$$

where, here and in the rest of the proof, $c$ denotes a generic (positive) constant independent of $k$. Also, by means of an integration by parts, we show

$$
\Re\left(\partial_{1} \boldsymbol{v}_{k}, \boldsymbol{v}_{k}^{*}\right)=0 .
$$

Thus, by taking the real part of (63) and using (64) and (65) we infer

$$
\left\|\nabla \boldsymbol{v}_{k}\right\|_{2}^{2} \leq c(|k|+1)\left\|\boldsymbol{v}_{k}\right\|_{2} .
$$

Likewise, taking the imaginary part of (63) and employing (64)-(66) along with Schwarz inequality, we obtain

$$
|k|\|\boldsymbol{v}\|_{2} \leq c\left(\left\|\nabla \boldsymbol{v}_{k}\right\|_{2}+|k|+1\right) \leq c(|k|+1)\left\|\boldsymbol{v}_{k}\right\|_{2}^{\frac{1}{2}},
$$

which implies

$$
\|\boldsymbol{v}\|_{2} \leq c
$$

Taking into account that $\boldsymbol{h}_{k}=\boldsymbol{\Phi}+\boldsymbol{v}_{k}$, (67) proves (59) ${ }_{1}$ for $\boldsymbol{h}_{k}$. Similarly, replacing (67) into (66), we arrive at $(59)_{2}$. Finally, from classical estimates on the Stokes problem [24] (Lemma 1) we find

$$
\left\|D^{2} \boldsymbol{v}_{k}\right\|_{2} \leq c\left(\left\|\partial_{1} \boldsymbol{v}\right\|_{2}+\left\|\mathcal{F}_{k}\right\|_{2}+\|\nabla \boldsymbol{v}\|_{2}\right)
$$

and so (59) $)_{3}$ follows from this inequality, (64), (66) and (67). Concerning the fields $\boldsymbol{H}_{k}^{(i)}$, let $\Psi^{(i)}=\phi(|x|) \boldsymbol{e}_{i} \times \boldsymbol{x}$, and set $\boldsymbol{V}_{k}^{(i)}:=\boldsymbol{H}^{(i)}+\Psi^{(i)}$. Obviously, the support of $\Psi^{(i)}$ is contained in $\Omega_{2 R}, \operatorname{div} \Psi^{(i)}=0$ and $\left.\Psi^{(i)}\right|_{\partial \Omega}=e_{i} \times x$. Thus, from (58) it follows that $\boldsymbol{V}_{k}^{(i)}$ is a solution to (62) with $\left(\boldsymbol{V}_{k}^{(i)}, \Psi^{(i)}\right)$ in place of $\left(\boldsymbol{v}_{k}, \boldsymbol{\Phi}\right)$. Therefore, we can use exactly the same arguments used earlier in the proof to show that also $\boldsymbol{H}_{k}^{(i)}$ satisfies the stated properties. Let $\alpha \in \mathbb{C}^{3}$, and, for fixed $k \neq 0$, set (Summation over repeated indices.)

$$
\mathbf{u}:=\alpha_{i} \boldsymbol{h}_{k}^{(i)}, \mathbf{q}:=\alpha_{i} p_{k}^{(i)}
$$

From (57) we then find

$$
\begin{aligned}
& \begin{array}{l}
\mathrm{i} k \mathbf{u}-\tau \partial_{1} \mathbf{u}=\operatorname{div} \mathbb{T}(\mathbf{u}, \mathbf{q}) \\
\operatorname{div} \mathbf{u}=0
\end{array} \\
& \mathbf{u}=\boldsymbol{\alpha} \text { at } \partial \Omega .
\end{aligned} \text { in } \Omega
$$


Dot-multiplying both sides of (68) 1 by $\mathbf{u}^{*}$ and integrating by parts over $\Omega$ we deduce

$$
ß k\|\mathbf{u}\|_{2}^{2}+\|\mathbb{D}(\mathbf{u})\|_{2}^{2}-\tau\left(\partial_{1} \mathbf{u}, \mathbf{u}^{*}\right)=\boldsymbol{\alpha}^{*} \cdot \mathbb{K} \cdot \boldsymbol{\alpha} .
$$

Now, suppose that there is $\hat{\boldsymbol{\alpha}} \in \mathbb{C}$ such that $\mathbb{K} \cdot \hat{\boldsymbol{\alpha}}=-\mathbb{\beta} \mu \hat{\boldsymbol{\alpha}}$, for some $\mu \in \mathbb{R}$. Then from the previous relation we obtain

$$
\mathbb{B}\left(k\|\mathbf{u}\|_{2}^{2}+\mu|\hat{\boldsymbol{\alpha}}|^{2}\right)-\tau\left(\partial_{1} \mathbf{u}, \mathbf{u}^{*}\right)=\|\mathbb{D}(\mathbf{u})\|_{2}^{2},
$$

which, in turn, taking into account that $\Re\left(\partial_{1} \mathbf{u}, \mathbf{u}^{*}\right)=0$, allows us to we deduce $\mathbf{u}=\mathbf{0}$ in $W^{2,2}(\Omega)$. The latter implies $\hat{\boldsymbol{\alpha}}=\mathbf{0}$ and thus shows the desired property for $\mathbb{K}$. In a similar manner, we prove the same property for $\mathbb{S}$. Next, let $\zeta \in \mathbb{C}^{6}$ and define (Summation over repeated indices.)

$$
\mathbf{v}:=\zeta_{i} \boldsymbol{h}_{k}^{(i)}+\zeta_{i+3} \boldsymbol{H}_{k}^{(i)}, \mathbf{p}:=\zeta_{i} p_{k}^{(i)}+\zeta_{i+3} P_{k}^{(i)} .
$$

Employing (57) and (58) we then deduce

$$
\begin{aligned}
& \left.\begin{array}{l}
\mathrm{i} k \mathbf{v}-\tau \partial_{1} \mathbf{v}=\operatorname{div} \mathbb{T}(\mathbf{v}, \mathrm{p}) \\
\operatorname{div} \mathbf{v}=0
\end{array}\right\} \text { in } \Omega \\
& \mathbf{v}=\zeta_{i} \boldsymbol{e}_{i}+\left(\zeta_{3+i} \boldsymbol{e}_{i}\right) \times x \text { at } \partial \Omega \text {. }
\end{aligned}
$$

By dot-multiplying both sides of $(70)_{1}$ by $\mathbf{v}^{*}$ and integrating by parts over $\Omega$, we show

$$
\mathrm{i} k\|\mathbf{v}\|_{2}^{2}+2\|\mathbb{D}(\mathbf{v})\|_{2}^{2}-\tau\left(\partial_{1} \mathbf{v}, \mathbf{v}^{*}\right)=\int_{\partial \Omega} \mathbf{v}^{*} \cdot \mathbb{T}(\mathbf{v}, \mathrm{p}) \cdot \boldsymbol{n} .
$$

As a consequence, (61) follows by replacing (69) in this last inequality and using (60) and (70) $)_{3}$. Finally, let $\zeta \in \mathbb{C}^{6}$ such that $(\boldsymbol{A}+\boldsymbol{g}) \cdot \boldsymbol{\zeta}=\mathbf{0}$. From (61) it then follows that

$$
\mathrm{i}\left[k\|\mathbf{v}\|_{2}^{2}+\sum_{i=1}^{3}\left(\lambda\left|\zeta_{i}\right|^{2}+\mu\left|\zeta_{i+3}\right|^{2}\right)\right]+2\|\mathbb{D}(\mathbf{v})\|_{2}^{2}-\tau\left(\partial_{1} \mathbf{v}, \mathbf{v}^{*}\right)=0,
$$

from which, recalling that $\Re\left(\partial_{1} \mathbf{v}, \mathbf{v}^{*}\right)=0$, and that $\mathbf{v}$ is a solution to (70) in the space $W^{1,2}(\Omega)$, we at once obtain $\mathbf{v} \equiv \mathbf{0}$, implying $\zeta=\mathbf{0}$. The proof is completed.

Remark 4. Even though the results of the previous lemma are stated for $\Omega$ the exterior of a ball, the reader will check with no effort that they continue to hold-without changes in their proof-for any exterior domain of class $C^{2}$. Therefore, they generalize those obtained in [25] (Lemma 5.1)

With the help of Lemma 7, we are now able to show the following one.

Lemma 8. Let $\tau \in \mathbb{R}$. Then, for any $(f, F, G) \in \mathcal{L}_{\sharp}^{2} \times L_{\sharp}^{2} \times L_{\sharp}^{2}$, the problem

$$
\begin{gathered}
\partial_{\mathrm{s}} \boldsymbol{w}-\boldsymbol{\tau} \cdot \nabla \boldsymbol{w}=\Delta \boldsymbol{w}-\nabla \phi+f \\
\operatorname{div} \boldsymbol{w}=0 \\
\boldsymbol{w}=\chi+\boldsymbol{\sigma} \times \boldsymbol{x} \text { at } \partial \Omega \times[0,2 \pi], \\
M \dot{\chi}+\int_{\partial \Omega} \mathbb{T}(\boldsymbol{w}, \phi) \cdot \boldsymbol{n}=\boldsymbol{F}, \quad \mathcal{I} \dot{\boldsymbol{\sigma}}+\int_{\partial \Omega} \boldsymbol{x} \times \mathbb{T}(\boldsymbol{w}, \phi) \cdot \boldsymbol{n}=\boldsymbol{G} \text { in }[0,2 \pi],
\end{gathered}
$$

has one and only one solution $(\boldsymbol{w}, \phi, \chi, \sigma) \in \mathcal{W}_{\sharp}^{2} \times \mathcal{P}^{1,2} \times W_{\sharp}^{1,2} \times W_{\sharp}^{1,2}$. This solution satisfies the estimate

$$
\|\boldsymbol{w}\|_{\mathcal{W}_{\sharp}^{2}}+\|\phi\|_{\mathcal{P}^{1,2}}+\|x\|_{W^{1,2}}+\|\boldsymbol{\sigma}\|_{W^{1,2}} \leq C_{2}\left(\|\boldsymbol{f}\|_{\mathcal{L}_{\sharp}^{2}}+\|\boldsymbol{F}\|_{L^{2}}+\|\boldsymbol{G}\|_{L^{2}}\right),
$$


where $C_{2}=C_{2}\left(\Omega, \tau, \rho_{\mathcal{S}} / \rho_{\mathcal{L}}\right)$.

Proof. Since the actual values of $M$ and $\mathcal{I}$ are irrelevant to the proof, we put, for simplicity, $M=\mathcal{I}=1$. Moreover, without loss of generality, we may take $\tau=\tau \boldsymbol{e}_{1}$. Let $\boldsymbol{w}=\boldsymbol{z}+\boldsymbol{u}$ where $z$ and $u$ satisfy the following set of equations

$$
\left.\begin{array}{l}
\partial_{\mathrm{s}} z-\tau \partial_{1} z-\Delta z=-\nabla \mathrm{r}+f \\
\operatorname{div} z=0 \\
\left.z\right|_{\partial \Omega}=\mathbf{0}
\end{array}\right\} \text { in } \Omega \times[0,2 \pi]
$$

and

$$
\begin{gathered}
\left.\begin{array}{c}
\partial_{\mathrm{s}} \boldsymbol{u}-\tau \partial_{1} u-\Delta u=-\nabla \mathrm{q} \\
\operatorname{div} \boldsymbol{u}=0
\end{array}\right\} \text { in } \Omega \times[0,2 \pi] \\
\left.\boldsymbol{u}\right|_{\partial \Omega}=\chi+\sigma \times x ; \\
\dot{\chi}+\int_{\partial \Omega} \boldsymbol{T}(\boldsymbol{u}, \mathrm{q}) \cdot \boldsymbol{n}=\boldsymbol{F}-\int_{\partial \Omega} \boldsymbol{T}(\boldsymbol{z}, \mathrm{r}) \cdot \boldsymbol{n}:=\mathcal{F}, \text { in }[0,2 \pi], \\
\dot{\sigma}+\int_{\partial \Omega} \boldsymbol{x} \times \mathbb{T}(\boldsymbol{u}, \mathrm{q}) \cdot \boldsymbol{n}=\boldsymbol{G}-\int_{\partial \Omega} \boldsymbol{x} \times \mathbb{T}(\boldsymbol{z}, \mathrm{r}) \cdot \boldsymbol{n}:=\mathcal{G}, \text { in }[0,2 \pi] .
\end{gathered}
$$

From [26] (Theorem 12), it follows that there exists a unique solution $(z, \tau) \in \mathcal{W}_{\sharp}^{2} \times \mathcal{P}^{1}$ that, in addition, obeys the inequality

$$
\|z\|_{\mathcal{W}_{\sharp}^{2}}+\|\mathrm{r}\|_{\mathcal{P}^{1,2}} \leq \mathrm{c}\|\boldsymbol{f}\|_{\mathcal{L}_{\sharp}^{2}} .
$$

Furthermore, by trace theorem (Possibly, by modifying $r$ by adding to it a suitable function of time.) and (75) we get

$$
\left\|\int_{\partial \Omega} \mathbb{T}(\boldsymbol{z}, \mathrm{r}) \cdot \boldsymbol{n}\right\|_{L^{2}}+\left\|\int_{\partial \Omega} \boldsymbol{x} \times \mathbb{T}(\boldsymbol{z}, \boldsymbol{\phi}) \cdot \boldsymbol{n}\right\|_{L^{2}} \leq c\left(\|\boldsymbol{z}\|_{\mathcal{W}_{\sharp}^{2}}+\|\mathrm{r}\|_{\mathcal{P}^{1,2}}\right) \leq c\|\boldsymbol{f}\|_{\mathcal{L}_{\sharp}^{2}},
$$

so that both functions $\mathcal{F}$ and $\mathcal{G}$ in $(74)$ are in $L_{\sharp}^{2}(0,2 \pi)$ and satisfy

$$
\|\mathcal{F}\|_{L^{2}}+\|\mathcal{G}\|_{L^{2}} \leq c\left(\|f\|_{\mathcal{L}_{\sharp}^{2}}+\|\boldsymbol{F}\|_{L^{2}}+\|G\|_{L^{2}}\right) .
$$

To find solutions to (74), we formally expand $u, q, \chi$ and $\sigma$ in Fourier series (summation over repeated indices):

$$
\begin{aligned}
& \boldsymbol{u}(x, \mathrm{~s})=\boldsymbol{u}_{k}(x) \mathrm{e}^{\Re k s}, \mathrm{q}(x, \mathrm{~s})=\mathrm{q}_{k}(x) \mathrm{e}^{\beta k \mathrm{~s}}, \chi(\mathrm{s})=\chi_{k} \mathrm{e}^{\Re k s}, \sigma(\mathrm{s})=\sigma_{k} \mathrm{e}^{\Re k s}, \\
& k \in \mathbb{Z} \backslash\{0\}, \boldsymbol{u}_{0} \equiv \nabla \mathrm{q}_{0} \equiv \chi_{0} \equiv \sigma_{0} \equiv \mathbf{0},
\end{aligned}
$$

where $\left(\boldsymbol{u}_{k}, \mathrm{q}_{k}, \boldsymbol{\chi}_{k}, \sigma_{k}\right)$ solve the problem $(k \neq 0)$

$$
\begin{gathered}
\begin{array}{l}
\otimes k \boldsymbol{u}_{k}-\tau \partial_{1} \boldsymbol{u}_{k}=\Delta \boldsymbol{u}_{k}-\nabla \mathrm{q}_{k} \\
\operatorname{div} \boldsymbol{u}_{k}=0
\end{array} \\
\left.\boldsymbol{u}_{k}\right|_{\partial \Omega}=\boldsymbol{\chi}_{k}+\sigma_{k} \times \boldsymbol{x},
\end{gathered} \text { in } \Omega
$$

subject to the further conditions

$$
ß k \chi_{k}+\int_{\partial \Omega} \mathbb{T}\left(\boldsymbol{u}_{k}, \mathrm{q}_{k}\right) \cdot \boldsymbol{n}=\mathcal{F}_{k}, B k \sigma_{k}+\int_{\partial \Omega} \boldsymbol{x} \times \mathbb{T}\left(\boldsymbol{u}_{k}, \mathrm{q}_{k}\right) \cdot \boldsymbol{n}=\mathcal{G}_{k},
$$


with $\left\{\mathcal{F}_{k}\right\},\left\{\mathcal{G}_{k}\right\}$ are Fourier coefficients of $\mathcal{F}$ and $\mathcal{G}$, respectively, and $\mathcal{F}_{0} \equiv \mathcal{G}_{0} \equiv \mathbf{0}$. For each fixed $k \in \mathbb{Z} \backslash\{0\}$, a solution to (78)-(79) is given by

$$
\boldsymbol{u}_{k}=\sum_{i=1}^{3}\left(\chi_{k i} \boldsymbol{n}_{k}^{(i)}+\sigma_{k i} \boldsymbol{H}_{k}^{(i)}\right), \mathbf{q}_{k}=\sum_{i=1}^{3}\left(\chi_{k i} p_{k}^{(i)}+\sigma_{k i} P_{k}^{(i)}\right)
$$

with $\left(\boldsymbol{h}_{k}^{(i)}, p_{k}^{(i)}\right),\left(\boldsymbol{H}_{k}^{(i)}, P_{k}^{(i)}\right)$ given in Lemma 7 , and where $\boldsymbol{\chi}_{k}, \sigma_{k}$ solve the equations

$$
\begin{aligned}
& B k \chi_{k}+\sum_{i=1}^{3} \int_{\partial \Omega}\left[\chi_{k i} \mathbb{T}\left(\boldsymbol{h}_{k}^{(i)}, p_{k}^{(i)}\right)+\sigma_{k i} \mathbb{T}\left(\boldsymbol{H}_{k}^{(i)}, P_{k}^{(i)}\right)\right] \cdot \boldsymbol{n}=\mathcal{F}_{k}, \\
& B k \sigma_{k}+\sum_{i=1}^{3} \int_{\partial \Omega}\left[\chi_{k i} \boldsymbol{x} \times \mathbb{T}\left(\boldsymbol{h}_{k}^{(i)}, p_{k}^{(i)}\right)+\sigma_{k i} x \times \mathbb{T}\left(\boldsymbol{H}_{k}^{(i)}, P_{k}^{(i)}\right)\right] \cdot \boldsymbol{n}=\mathcal{G}_{k} .
\end{aligned}
$$

Set

$$
\xi_{k}:=\left(\chi_{k}, \sigma_{k}\right) \in \mathbb{C}^{6}, \mathcal{F}_{k}:=\left(\mathcal{F}_{k}, \mathcal{G}_{k}\right) \in \mathbb{C}^{6} .
$$

Then, with the notation of Lemma 7 , (81) can be equivalently rewritten as

$$
\mathcal{B} \cdot \mathcal{\xi}_{k}=\mathcal{F}_{k},
$$

where $\mathcal{B}:=\mathbb{B} k \boldsymbol{I}+\mathcal{A}$, and $\boldsymbol{I}$ is the $6 \times 6$ identity matrix. By that lemma the matrix $\mathcal{B}$ is invertible for all $k \in \mathbb{Z}$. Furthermore, from (61), for all $\zeta \in \mathbb{C}^{6}$ we get

$$
\zeta^{*} \cdot \boldsymbol{B} \cdot \zeta=ß k\left(|\zeta|^{2}+\|\mathbf{v}\|_{2}^{2}\right)-\tau\left(\partial_{1} \mathbf{v}, \mathbf{v}^{*}\right)+2\|\mathbb{D}(\mathbf{v})\|_{2}^{2} .
$$

As a result, for any given $\mathcal{F}_{k},(82)$ has one and only one solution $\xi_{k}$.

We next dot-multiply both sides of (82) by $\xi_{k}^{*}$ and use (83) to deduce

$$
B k\left(\left|\boldsymbol{\xi}_{k}\right|^{2}+\left\|\boldsymbol{u}_{k}\right\|_{2}^{2}\right)-\tau\left(\partial_{1} \boldsymbol{u}_{k}, \boldsymbol{u}_{k}^{*}\right)+2\left\|\mathbb{D}\left(\boldsymbol{u}_{k}\right)\right\|_{2}^{2}=\left(\mathcal{F}_{k}, \boldsymbol{\xi}_{k}^{*}\right),
$$

which, by Cauchy-Schwarz inequality and (16) furnishes, in particular, the following estimates for all $k \in \mathbb{Z} \backslash\{0\}$

$$
\begin{aligned}
& \left\|\mathbb{D}\left(\boldsymbol{u}_{k}\right)\right\|_{2}^{2} \leq \frac{1}{2}\left\|\mathcal{F}_{k}\right\|_{2}\left|\boldsymbol{\xi}_{k}\right| \\
& |k|\left\|\boldsymbol{u}_{k}\right\|_{2}^{2} \leq 2|\tau|^{2}\left\|\nabla \boldsymbol{u}_{k}\right\|_{2}^{2}+2\left\|\mathcal{F}_{k}\right\|_{2}\left|\xi_{k}\right| \leq 4|\tau|^{2}\left\|\mathbb{D}\left(\boldsymbol{u}_{k}\right)\right\|_{2}^{2}+2\left\|\mathcal{F}_{k}\right\|_{2}\left|\boldsymbol{\xi}_{k}\right| \\
& |k|\left|\boldsymbol{\xi}_{k}\right|^{2} \leq 2|\tau|\left\|\nabla \boldsymbol{u}_{k}\right\|_{2}\left\|\boldsymbol{u}_{k}\right\|_{2}+\frac{2}{|k|}\left\|\boldsymbol{F}_{k}\right\|_{2}^{2} \leq 2^{\frac{3}{2}}|\tau|\left\|\mathbb{D}\left(\boldsymbol{u}_{k}\right)\right\|_{2}\left\|\boldsymbol{u}_{k}\right\|_{2}+\frac{2}{|k|}\left\|\mathcal{F}_{k}\right\|_{2}^{2}
\end{aligned}
$$

Replacing $(84)_{1}$ into $(84)_{2}$, we obtain

$$
|k|\left\|\boldsymbol{u}_{k}\right\|_{2}^{2} \leq c\left\|\mathcal{F}_{k}\right\|_{2}\left|\boldsymbol{\xi}_{k}\right|,
$$

while using $(84)_{1}$ and (85) into $(84)_{3}$ along with Cauchy-Schwarz inequality implies

$$
|k|^{2}\left|\boldsymbol{\xi}_{k}\right|_{2}^{2} \leq c\left\|\mathcal{F}_{k}\right\|_{2}^{2} .
$$

Combining (84) $)_{1}-(86)$ and (16), and recalling (59) 3 and (80) we thus infer

$$
\sum_{|k| \geq 1}\left[\left(|k|^{2}+1\right)\left\|\boldsymbol{u}_{k}\right\|_{2}^{2}+\left\|\nabla \boldsymbol{u}_{k}\right\|_{2}^{2}+\left\|D^{2} \boldsymbol{u}_{k}\right\|_{2}^{2}\right] \leq c \sum_{|k| \geq 1}\left(|k|^{2}+1\right)\left|\boldsymbol{\xi}_{k}\right|^{2} \leq c\|\mathcal{F}\|_{L^{2}}^{2} .
$$


Therefore, we may conclude that the quadruple $\left(\boldsymbol{u}, \mathrm{q}, \chi_{\boldsymbol{u}} \equiv \chi, \sigma_{\boldsymbol{u}} \equiv \boldsymbol{\sigma}\right)$ defined in (77) with $\left(\boldsymbol{u}_{k}, \mathrm{q}_{k}, \boldsymbol{\chi}_{k}, \sigma_{k}\right)$ satisfying (78)-(79) is a solution to (74) in the class $\mathcal{W}_{\sharp}^{2} \times \mathcal{P}^{1,2} \times W_{\sharp}^{1,2} \times$ $W_{\sharp}^{1,2}$. Furthermore, (76) and (87) also entail the validity of the following inequality

$$
\|\boldsymbol{u}\|_{\mathcal{W}_{\sharp}^{2}}+\|\mathrm{q}\|_{\mathcal{P}^{1,2}}+\|\boldsymbol{\chi}\|_{W^{1,2}}+\|\boldsymbol{\sigma}\|_{W^{1,2}} \leq c\left(\|\boldsymbol{f}\|_{\mathcal{L}_{\sharp}^{2}}+\|\boldsymbol{F}\|_{L^{2}}+\|\boldsymbol{G}\|_{L^{2}}\right) .
$$

The existence proof is thus completed. The uniqueness property amounts to show that the problem

$$
\begin{aligned}
& \left.\begin{array}{l}
\partial_{\mathrm{s}} \boldsymbol{w}-\tau \partial_{1} \boldsymbol{w}=\Delta \boldsymbol{w}-\nabla \mathrm{p} \\
\operatorname{div} \boldsymbol{w}=0
\end{array}\right\} \text { in } \Omega \times[0,2 \pi] \\
& \left.w\right|_{\partial \Omega}=\chi+\sigma ; \\
& \dot{\chi}+\int_{\partial \Omega} \mathbb{T}(w, \mathrm{p}) \cdot \boldsymbol{n}=\mathbf{0}, \quad \dot{\sigma}+\int_{\partial \Omega} x \times \mathbb{T}(w, \mathrm{p}) \cdot \boldsymbol{n}=\mathbf{0}
\end{aligned}
$$

has only the zero solution in the specified function class. If we dot-multiply $(88)_{1}$ by $w$, integrate by parts over $\Omega$ and use (88) 3 , we get

$$
\frac{1}{2} \frac{d}{d t}\left(\|\boldsymbol{w}(t)\|_{2}^{2}+|\chi(t)|^{2}+|\sigma(t)|^{2}\right)+2\|\mathbb{D}(\boldsymbol{w}(t))\|_{2}^{2}=0 .
$$

Integrating both sides of this equation from 0 to $2 \pi$ and employing the $2 \pi$-periodicity of the solution we easily obtain $\|\mathbb{D}(w(t))\|_{2} \equiv 0$ which, in turn, by the characterization of the space $\mathcal{V}$ given in Lemma 3, immediately furnishes $\boldsymbol{w} \equiv \nabla \mathrm{p} \equiv \mathbf{0}$. The proof of the lemma is completed.

Remark 5. Concerning the generality of the domain $\Omega$, an observation similar to that made in Remark 4 for Lemma 7, equally applies also to Lemma 8.

Let $\mathcal{F} \in \mathfrak{H}_{\sharp}$ where

$$
\mathcal{F}=\left\{\begin{array}{l}
f \text { in } \Omega \\
F+G \times x \text { in } \Omega_{0}
\end{array},\right.
$$

and consider the operator equation

$$
\mathcal{Q}_{0}(\boldsymbol{w})=\mathcal{F}
$$

By Lemma 2, (89) is equivalent to the following problem (with $\tau:=\lambda_{\mathrm{c}} \tau_{\mathrm{c}}, \chi:=\hat{\boldsymbol{w}}_{1}$, $\left.\sigma:=\hat{w}_{2}\right)$

$$
\begin{gathered}
\zeta_{0} \partial_{\mathrm{s}} \boldsymbol{w}-\lambda_{\mathrm{c}} \boldsymbol{\tau}_{\mathrm{c}} \cdot \nabla \boldsymbol{w}-\Delta \boldsymbol{w}=\nabla \phi+f \\
\operatorname{div} \boldsymbol{w}=0 \\
\boldsymbol{w}=\boldsymbol{\chi}+\boldsymbol{\sigma} \times \boldsymbol{x} \text { at } \partial \Omega \times[0,2 \pi] \\
M \dot{\chi}+\int_{\partial \Omega} \mathbb{T}(\boldsymbol{w}, \phi) \cdot \boldsymbol{n}-\boldsymbol{F}+\left(\mathcal{I} \dot{\boldsymbol{\sigma}}+\int_{\partial \Omega} \boldsymbol{y} \times \mathbb{T}(\boldsymbol{w}, \phi) \cdot \boldsymbol{n}-\boldsymbol{G}\right) \times \boldsymbol{x}=\mathbf{0} \text { in } \Omega_{0} \times[0,2 \pi] .
\end{gathered}
$$

Since $x$ is arbitrary in $\Omega_{0}$, we conclude that $(90)_{4,5}$ are equivalent to $(71)_{4,5}$. Thus, in view of Lemma 8, we deduce the following important result

Lemma 9. The operator $\mathcal{Q}_{0}$ is a homeomorphism.

This lemma allows us to prove the following theorem that represents the main result of this section. 
Theorem 5. The operator $\mathcal{Q}$ is Fredholm of index 0.

Proof. We commence to notice that $\mathcal{Q}=\mathcal{Q}_{0}+\mathcal{P} \mathcal{K}$. Thus, by Lemma 8 , the stated property will follow, provided we show that the map

$$
\mathcal{C}: w \in \mathfrak{W}_{\sharp}^{2} \mapsto \mathcal{K}(w) \in \mathfrak{L}_{\sharp}^{2}
$$

is compact. Let $\left\{\boldsymbol{w}_{k}\right\}$ be a bounded sequence in $\mathfrak{W}_{\sharp}^{2}$. This implies, in particular, that there is $M>0$ independent of $k$ such that $\left.\left(\chi_{k}:=\widehat{\left(\boldsymbol{w}_{k}\right)}\right)_{1}\right)$

$$
\left\|\boldsymbol{w}_{k}\right\|_{\mathcal{W}_{\sharp}^{2}}+\left\|\chi_{k}\right\|_{W^{1,2}} \leq M \text {. }
$$

We may then select sequences (again denoted by $\left.\left\{\boldsymbol{w}_{k}, \chi_{k}\right\}\right)$ and find $\left(\boldsymbol{w}_{*}, \boldsymbol{\chi}_{*}\right) \in$ $\mathcal{W}_{\sharp}^{2} \times W_{\sharp}^{1,2}$ such that

$$
\boldsymbol{w}_{k} \rightarrow \boldsymbol{w}_{*} \text { weakly in } \mathcal{W}_{\sharp}^{2} ; \chi_{k} \rightarrow \chi_{*} \text { strongly in } L^{\infty}(0,2 \pi) \text {. }
$$

Without loss of generality, we may take $w_{*} \equiv \chi_{*} \equiv \mathbf{0}$. We then have to show that

$$
\lim _{k \rightarrow \infty} \int_{0}^{T}\left\|\mathcal{K}\left(\boldsymbol{w}_{k}\right)\right\|_{2, \Omega}^{2}=0
$$

From (92), the compact embeddings $W^{2,2}(\Omega) \subset W^{1,4}\left(\Omega_{R}\right) \subset L^{2}\left(\Omega_{R}\right)$ for all $R>1$, and Lions-Aubin lemma we then have

$$
\int_{0}^{2 \pi}\left(\left\|\boldsymbol{w}_{k}(t)\right\|_{2, \Omega_{R}}^{2}+\left\|\nabla \boldsymbol{w}_{k}(t)\right\|_{2, \Omega_{R}}^{2}\right) \mathrm{d} t \rightarrow 0 \text { as } k \rightarrow \infty, \text { for all } R>1 .
$$

Further, by (52),

$$
\int_{0}^{2 \pi}\left\|\boldsymbol{v}_{\mathrm{c}} \cdot \nabla \boldsymbol{w}_{k}(t)\right\|_{2, \Omega}^{2} \leq\left\|\boldsymbol{v}_{\mathrm{c}}\right\|_{\infty}^{2} \int_{0}^{2 \pi}\left\|\nabla \boldsymbol{w}_{k}(t)\right\|_{2, \Omega_{R}}^{2}+\left\|\boldsymbol{v}_{\mathrm{c}}\right\|_{4, \Omega^{R}}^{2} \int_{-\pi}^{\pi}\left\|\nabla \boldsymbol{w}_{k}(t)\right\|_{4, \Omega}^{2},
$$

which, by (52) $1,(91),(94)$ and the arbitrariness of $R$ furnishes

$$
\lim _{k \rightarrow \infty} \int_{0}^{2 \pi}\left\|\boldsymbol{v}_{\mathrm{c}} \cdot \nabla \boldsymbol{w}_{k}(\tau)\right\|_{2}^{2}=0 .
$$

Likewise,

$$
\int_{0}^{2 \pi}\left\|\boldsymbol{w}_{k}(t) \cdot \nabla \boldsymbol{v}_{\mathrm{c}}\right\|_{2, \Omega}^{2} \leq\left\|\nabla \boldsymbol{v}_{\mathrm{c}}\right\|_{\infty}^{2} \int_{0}^{2 \pi}\left\|\boldsymbol{w}_{k}(t)\right\|_{2, \Omega_{R}}^{2}+\left\|\nabla \boldsymbol{v}_{\mathrm{c}}\right\|_{2, \Omega^{R}}^{2} \int_{0}^{\pi}\left\|\boldsymbol{w}_{k}(t)\right\|_{2, \Omega}^{2},
$$

so that, by (52) $2,(92)_{1}$, and (94) we deduce, as before,

$$
\lim _{k \rightarrow \infty} \int_{0}^{2 \pi}\left\|\boldsymbol{w}_{k}(t) \cdot \nabla \boldsymbol{v}_{\mathrm{c}}\right\|_{2, \Omega}^{2}=0
$$

Finally,

$$
\int_{0}^{2 \pi}\left\|\chi_{k} \cdot \nabla v_{\mathrm{c}}\right\|_{2, \Omega}^{2} \leq 2 \pi\left\|\chi_{k}\right\|_{L^{\infty}(0,2 \pi)}^{2}\left\|\nabla v_{\mathrm{c}}\right\|_{2}^{2}
$$

which, by (52) 2 and (92) 2 furnishes

$$
\lim _{k \rightarrow \infty} \int_{0}^{2 \pi}\left\|\chi_{k} \cdot \nabla v_{\mathrm{c}}\right\|_{2, \Omega}^{2}=0
$$

Combining (95)-(97) we thus arrive at (93), which completes the proof of the theorem. 


\section{Sufficient Conditions for Time-Periodic Bifurcation}

The first objective of this section is to rewrite (4) in an operator form of the type (9) and then, successively, employ Theorem 1 to provide sufficient conditions for the occurence of time-periodic bifurcation for our problem. Thus, let

$$
\boldsymbol{u}(x, \mathrm{~s})=\overline{\boldsymbol{u}}(x)+\boldsymbol{w}(x, \mathrm{~s}), \mathrm{p}=\overline{\mathrm{p}}(x)+\phi(x, \mathrm{~s}), \gamma=\bar{\gamma}+\chi(\mathrm{s}), \boldsymbol{\omega}(\mathrm{s})=\overline{\boldsymbol{\omega}}+\boldsymbol{\sigma}(\mathrm{s}) .
$$

Then, (4) can be equivalently written in terms of the two sets of unknowns $(\overline{\boldsymbol{u}}, \overline{\mathrm{p}}, \bar{\gamma}, \overline{\boldsymbol{\omega}})$ and $(\boldsymbol{w}, \phi, \chi, \sigma)$ as follows

$$
\begin{gathered}
\begin{array}{l}
-\lambda \boldsymbol{\tau} \cdot \nabla \overline{\boldsymbol{u}}+\lambda(\boldsymbol{v} \cdot \nabla \overline{\boldsymbol{u}}+(\overline{\boldsymbol{u}}-\bar{\gamma}) \cdot \nabla \boldsymbol{v})+\lambda(\overline{\boldsymbol{u}}-\bar{\gamma}) \cdot \nabla \overline{\boldsymbol{u}}+\lambda \overline{(\boldsymbol{w}-\chi) \cdot \nabla \boldsymbol{w}}=\Delta \overline{\boldsymbol{u}}-\nabla \overline{\mathrm{p}} \\
\operatorname{div} \overline{\boldsymbol{u}}=0
\end{array} \\
\overline{\boldsymbol{u}}=\bar{\gamma}+\overline{\boldsymbol{w}} \times \boldsymbol{x} \text { at } \partial \Omega, \lim _{|\boldsymbol{x}| \rightarrow \infty} \overline{\boldsymbol{u}}(x)=\mathbf{0}, \\
\int_{\partial \Omega} \mathbb{T}(\overline{\boldsymbol{u}}, \overline{\mathrm{p}}) \cdot \boldsymbol{n}=\mathbf{0}, \quad \int_{\partial \Omega} \boldsymbol{x} \times \mathbb{T}(\overline{\boldsymbol{u}}, \overline{\mathrm{p}}) \cdot \boldsymbol{n}=\mathbf{0},
\end{gathered}
$$

and

$$
\begin{aligned}
& \left.\begin{array}{l}
\zeta \partial_{s} w-\lambda \tau \cdot \nabla w+\lambda(v \cdot \nabla w+(w-\chi) \cdot \nabla v)+\lambda[(w-\chi) \cdot \nabla \bar{u} \\
+(\bar{u}-\bar{\gamma}) \cdot \nabla w+(w-\chi) \cdot \nabla w-\overline{(w-\chi) \cdot \nabla w}]=\Delta w-\nabla \phi \\
\operatorname{div} w=0
\end{array}\right\} \text { in } \Omega \times[0,2 \pi], \\
& w=\chi+\sigma \times x \text { at } \partial \Omega \times[0,2 \pi], \quad \lim _{|x| \rightarrow \infty} w(x, \mathrm{~s})=0, \mathrm{~s} \in[0,2 \pi], \\
& \zeta M \dot{\chi}+\int_{\partial \Omega} \mathbb{T}(\boldsymbol{w}, \phi) \cdot \boldsymbol{n}=\mathbf{0}, \zeta \mathcal{I} \dot{\boldsymbol{\sigma}}+\int_{\partial \Omega} \boldsymbol{x} \times \mathbb{T}(\boldsymbol{w}, \phi) \cdot \boldsymbol{n}=\mathbf{0} \text { in }[0,2 \pi] .
\end{aligned}
$$

where, for simplicity, we have suppressed the subscript 0 . Let $v_{c}$ be the weak solution to (3) at $\lambda=\lambda_{\mathrm{c}}$, and let $\tau_{\mathrm{c}}$ be the associated translational velocity. We make the assumption that both $\tau$ and $\bar{\gamma}$ are directed along the direction $e_{\mathrm{c}}:=\tau_{\mathrm{c}} /\left|\tau_{\mathrm{c}}\right|$, and write $\tau=\tau \boldsymbol{e}_{\mathrm{c}}$, $\bar{\gamma}=\bar{\gamma} e_{\mathrm{c}}$. As a result, by Theorem 3 we know that, under the hypothesis (38), at $\lambda=\lambda_{\mathrm{c}}$ there exists an analytic family of weak solutions $v=v(\lambda)$ such that $v\left(\lambda_{\mathrm{c}}\right)=v_{\mathrm{c}}$. Thus, setting $\mu:=\lambda-\lambda_{c}$, (98) can be rewritten as follows

$$
\left.\begin{array}{c}
-\Delta \overline{\boldsymbol{u}}-\lambda_{\mathrm{c}} \tau_{\mathrm{c}} \boldsymbol{e}_{\mathrm{c}} \cdot \nabla \overline{\boldsymbol{u}}+\lambda_{\mathrm{c}}\left(\boldsymbol{v}_{\mathrm{c}} \cdot \nabla \overline{\boldsymbol{u}}+\left(\overline{\boldsymbol{u}}-\bar{\gamma} \boldsymbol{e}_{\mathrm{c}}\right) \cdot \nabla \boldsymbol{v}_{\mathrm{c}}\right)=\boldsymbol{N}_{1}(\overline{\boldsymbol{u}}, \boldsymbol{w}, \boldsymbol{\mu})-\nabla \overline{\mathrm{p}} \\
\operatorname{div} \overline{\boldsymbol{u}}=0
\end{array}\right\} \text { in } \Omega,
$$

where

$$
\begin{aligned}
\boldsymbol{N}_{1}(\overline{\boldsymbol{u}}, \boldsymbol{w}, \mu):= & -\left[\left(\widetilde{\mu} \widetilde{\tau}(\mu)-\lambda_{\mathrm{c}} \tau_{\mathrm{c}}\right) \boldsymbol{e}_{\mathrm{c}}+\widetilde{\mu} \widetilde{\boldsymbol{v}}(\mu)-\lambda_{c} \boldsymbol{v}_{\mathrm{c}}\right] \cdot \nabla \overline{\boldsymbol{u}} \\
& -\lambda_{\mathrm{c}}\left(\overline{\boldsymbol{u}}-\bar{\gamma} \boldsymbol{e}_{\mathrm{c}}\right) \cdot \nabla\left(\widetilde{\boldsymbol{v}}(\mu)-\boldsymbol{v}_{\mathrm{c}}\right)-\mu\left(\overline{\boldsymbol{u}}-\bar{\gamma} \boldsymbol{e}_{\mathrm{c}}\right) \cdot \nabla \widetilde{\boldsymbol{v}}(\mu) \\
& -\widetilde{\boldsymbol{\mu}}\left[\left(\overline{\boldsymbol{u}}-\bar{\gamma} \boldsymbol{e}_{\mathrm{c}}\right) \cdot \nabla \overline{\boldsymbol{u}}+\overline{(\boldsymbol{w}-\chi) \cdot \nabla \boldsymbol{w}}\right],
\end{aligned}
$$

and $\widetilde{f}(\mu):=f\left(\mu+\lambda_{c}\right)$. Likewise, (99) can be rewritten as follows

$$
\begin{aligned}
& \left.\begin{array}{l}
\zeta \partial_{\mathrm{s}} w-\lambda_{\mathrm{c}} \tau_{\mathrm{c}} \boldsymbol{e}_{\mathrm{c}} \cdot \nabla w+\lambda_{\mathrm{c}}\left(\boldsymbol{v}_{\mathrm{c}} \cdot \nabla w+(w-\chi) \cdot \nabla v_{\mathrm{c}}\right) \\
-\Delta w=N_{2}(\bar{u}, w, \mu)-\nabla \phi \\
\operatorname{div} \boldsymbol{w}=0
\end{array}\right\} \text { in } \Omega \times[0,2 \pi], \\
& w=\chi+\sigma \times x \text { at } \partial \Omega \times[0,2 \pi], \lim _{|x| \rightarrow \infty} w(x, \mathrm{~s})=\mathbf{0}, \mathrm{s} \in[0,2 \pi], \\
& \zeta M \dot{\chi}+\int_{\partial \Omega} \mathbb{T}(\boldsymbol{w}, \phi) \cdot \boldsymbol{n}=\mathbf{0}, \zeta \mathcal{I} \dot{\boldsymbol{\sigma}}+\int_{\partial \Omega} \boldsymbol{x} \times \mathbb{T}(\boldsymbol{w}, \phi) \cdot \boldsymbol{n}=\mathbf{0} \text { in }[0,2 \pi],
\end{aligned}
$$


where

$$
\begin{aligned}
\boldsymbol{N}_{2}(\overline{\boldsymbol{u}}, \boldsymbol{w}, \mu):= & -\left[\left(\widetilde{\mu} \widetilde{\tau}(\mu)-\lambda_{\mathrm{c}} \tau_{\mathrm{c}}\right) \boldsymbol{e}_{\mathrm{c}}+\widetilde{\mu} \widetilde{\boldsymbol{v}}(\mu)-\lambda_{c} \boldsymbol{v}_{\mathrm{c}}\right] \cdot \nabla \boldsymbol{w}-\lambda_{\mathrm{c}}(\boldsymbol{w}-\chi) \\
& \cdot \nabla\left(\widetilde{\boldsymbol{v}}(\mu)-\boldsymbol{v}_{\mathrm{c}}\right)-\mu(\boldsymbol{w}-\chi) \cdot \nabla \widetilde{\boldsymbol{v}}(\mu)-\widetilde{\mu}[(\boldsymbol{w}-\chi) \cdot \nabla \overline{\boldsymbol{u}} \\
& +(\overline{\boldsymbol{u}}-\bar{\gamma}) \cdot \nabla \boldsymbol{w}+(\boldsymbol{w}-\chi) \cdot \nabla \boldsymbol{w}-\overline{(\boldsymbol{w}-\chi) \cdot \nabla \boldsymbol{w}}]
\end{aligned}
$$

Let

$$
\widetilde{N}_{2}=\left\{\begin{array}{l}
N_{2} \text { in } \Omega \\
0 \text { in } \Omega_{0}
\end{array} .\right.
$$

The following result holds.

Lemma 10. The operators

$$
\begin{aligned}
& \mathcal{N}_{1}:(\bar{u}, \boldsymbol{w}, \mu) \in \mathcal{X}_{\mathrm{c}}\left(\mathbb{R}^{3}\right) \times \mathfrak{W}_{\sharp}^{2} \times \mathbb{R} \mapsto \boldsymbol{N}_{1}(\overline{\boldsymbol{u}}, \boldsymbol{w}, \mu) \in \mathcal{V}^{-1}\left(\mathbb{R}^{3}\right) \\
& \widetilde{\mathcal{N}}_{2}:(\overline{\boldsymbol{u}}, \boldsymbol{w}, \mu) \in \mathcal{X}_{\mathrm{c}}\left(\mathbb{R}^{3}\right) \times \mathfrak{W}_{\sharp}^{2} \times \mathbb{R} \mapsto \widetilde{\boldsymbol{N}}_{2}(\overline{\boldsymbol{u}}, \boldsymbol{w}, \mu) \in \mathfrak{L}_{\sharp}^{2}
\end{aligned}
$$

are well defined.

Proof. Since $\overline{\boldsymbol{u}}, \boldsymbol{v}, \boldsymbol{v}_{\mathrm{c}} \in \mathcal{X}_{\mathrm{c}}$, it follows at once that $\boldsymbol{e}_{\mathrm{c}} \cdot \nabla \overline{\boldsymbol{u}}, \boldsymbol{e}_{\mathrm{c}} \cdot \nabla \boldsymbol{v}, \boldsymbol{e}_{\mathrm{c}} \cdot \nabla \boldsymbol{v}_{\mathrm{c}} \in \mathcal{V}^{-1}$. Moreover, by Lemma 4 we also have $\overline{\boldsymbol{u}}, \boldsymbol{v}, \boldsymbol{v}_{\mathrm{c}} \in L^{4}\left(\mathbb{R}^{3}\right)$, which, by using integration by parts, implies $\boldsymbol{v} \cdot \nabla \overline{\boldsymbol{u}}, \boldsymbol{v}_{\mathrm{C}} \cdot \nabla \overline{\boldsymbol{u}}, \overline{\boldsymbol{u}} \cdot \nabla \boldsymbol{v}, \overline{\boldsymbol{u}} \cdot \nabla \boldsymbol{v}_{\mathrm{c}}, \overline{\boldsymbol{u}} \cdot \nabla \overline{\boldsymbol{u}} \in \mathcal{V}^{-1}\left(\mathbb{R}^{3}\right)$ as well. Finally, observing that $\boldsymbol{w} \in$ $L^{2}\left(L^{2} \cap L^{4}\right)$ and $\chi \in L^{\infty}(0,2 \pi)$ we easily show that $\overline{(\boldsymbol{w}-\chi) \cdot \nabla \boldsymbol{w}} \in \mathcal{V}^{-1}$, which concludes the proof of (104) 1 . By known embedding theorems [27] (Theorem 2.1) it follows that $w \in L^{2}\left(D^{1,4}\right)$. Thus, the validity of $(104)_{2}$ can be established along the same lines used to show $(104)_{1}$. We will omit the details. The proof of the lemma is completed.

In view of Lemma 10, and (36), (37), and (56), it follows at once that, setting $\mathcal{N}_{2}=\mathcal{P} \widetilde{\mathcal{N}}_{2}$, the coupled problem (100)-(103) can be written as operator equations:

$$
\begin{aligned}
& \mathcal{L}_{1}(\overline{\boldsymbol{u}})=\mathcal{N}_{1}(\overline{\boldsymbol{u}}, \boldsymbol{w}, \mu) \text { in } \mathcal{V}^{-1}\left(\mathbb{R}^{3}\right), \\
& \zeta \partial_{\mathbf{s}} \boldsymbol{w}+\mathcal{L}_{2}(\boldsymbol{w})=\mathcal{N}_{2}(\overline{\boldsymbol{u}}, \boldsymbol{w}, \mu) \text { in } \mathfrak{L}_{\sharp}^{2}
\end{aligned}
$$

which are in the form (9). We shall next check how the assumptions of Theorem 1 can be satisfied in our case. We begin to notice that Theorem 5 secures (H3). In addition, both hypotheses (H1) and (H4) are verified if we assume

$$
\mathrm{N}\left[\mathcal{L}_{1}\right]=\{\mathbf{0}\} .
$$

In fact, by Theorem $3, \mathcal{L}_{1}$ is Fredholm of index 0 , so that (106) implies (H1). Moreover, if (106) holds, then -taking into account that the nonlinear operators $\mathcal{N}_{i}, i=1,2$, are (at most) quadratic in $(\overline{\boldsymbol{u}}, \boldsymbol{w})$ - again by Theorem 3 , we deduce the validity of (H4). Next, we assume

$v_{0}:=\beta \zeta_{0}, \zeta_{0} \neq 0$, is a simple eigenvalue of $\mathcal{L}_{2}$, and $k v_{0} \in \mathrm{P}\left(\mathcal{L}_{2}\right)$, for all $k \in \mathbb{N} \backslash\{0,1\}$.

This, in view of Theorem 4, guarantees assumption (H2). By a straightforward calculation, we can sho that, in our case, the operator $S_{011}$ is given by

$$
\left(\tau_{c} e_{c}+v_{c}\right) \cdot \nabla w+\lambda_{c}\left(\tau^{\prime}\left(\lambda_{c}\right) e_{c}+v^{\prime}\left(\lambda_{c}\right)\right) \cdot \nabla w+(w-\chi) \cdot \nabla\left(v_{c}+v^{\prime}\left(\lambda_{c}\right)\right),
$$

where the prime means differentiation with respect to $\mu$. So, denoting by $v=v(\mu)$ the eigenvalues of $\mathcal{L}_{2}+\mu S_{011}$, by [14] (Proposition 79.15 and Corollary 79.16)) we have that in a neighborhood of $\mu=0$ the map $\mu \mapsto v(\mu)$ is well defined and of class $C^{\infty}$. This justifies our last assumption:

$$
\Re\left[v^{\prime}(0)\right] \neq 0 .
$$


We are now in a position to employ Theorem 1 to obtain the following main result.

Theorem 6. Suppose (106)-(108) hold. Let $\boldsymbol{w}_{0}$ be the normalized eigenvector of $\mathcal{L}_{2}$ corresponding to the eigenvalue $v_{0}$, and set $w_{1}:=\Re\left[w_{0} \mathrm{e}^{-\mathrm{i} s}\right]$. Then, the following properties are valid.

(a) Existence. There are analytic families

$$
(\overline{\boldsymbol{u}}(\varepsilon), \boldsymbol{w}(\varepsilon), \zeta(\varepsilon), \mu(\varepsilon)) \in X_{c} \times \mathfrak{W}_{\sharp}^{2} \times \mathbb{R}_{+} \times \mathbb{R}
$$

satisfying (105), for all real $\varepsilon$ in a neighborhood $\mathcal{I}(0)$ of 0 , and such that

$$
\left(\overline{\boldsymbol{u}}(\varepsilon), \boldsymbol{w}(\varepsilon)-\varepsilon \boldsymbol{w}_{1}, \zeta(\varepsilon), \mu(\varepsilon)\right) \rightarrow\left(0,0, \zeta_{0}, 0\right) \text { as } \varepsilon \rightarrow 0 .
$$

(b) Uniqueness. There is a neighborhood

$$
U\left(0,0, \zeta_{0}, 0\right) \subset X_{c} \times \mathfrak{W}_{\sharp}^{2} \times \mathbb{R}_{+} \times \mathbb{R}
$$

such that every (nontrivial) $2 \pi$-periodic solution to (105), lying in $U$ must coincide, up to a phase shift, with a member of the family (109).

(c) Parity. The functions $\zeta(\varepsilon)$ and $\mu(\varepsilon)$ are even:

$$
\zeta(\varepsilon)=\zeta(-\varepsilon), \quad \mu(\varepsilon)=\mu(-\varepsilon), \text { for all } \varepsilon \in \mathcal{I}(0) .
$$

Consequently, the bifurcation due to these solutions is either subcritical or supercritical, a two-sided bifurcation being excluded (Unless $\mu \equiv 0$ ).

\section{On the Motion of the Sphere in the Time-Periodic Regime}

As we mentioned in the introductory section, experimental and numerical tests show that, in the transition from steady to time-periodic motion, the trajectory of the center of mass, $G$, of the sphere changes from a rectilinear to a zigzag path. Objective of this section is to study in more details the motion of the sphere in the time-periodic regime and, in particular, to derive necessary and sufficient conditions for the occurrence of this sideway oscillatory behavior.

To this end, we begin to observe that, according to Theorem 6, in the neighborhood of $\lambda_{c}$, namely, for sufficiently small $\varepsilon$, the oscillatory part of the solution, $w$, behaves like the corresponding solution to the linear problem $\mathcal{Q}\left(\boldsymbol{w}_{1}\right)=\mathbf{0}$, that is, $\boldsymbol{w}_{1}:=\Re\left[\boldsymbol{w}_{0} \mathrm{e}^{-\mathrm{is}}\right]$, with $w_{0}$ eigenvector of $\mathcal{L}_{2}$ corresponding to the eigenvalue $-\beta \zeta_{0}$. Therefore, in such a neighborhood, the oscillatory component of the velocity of $G$ will have the same kinematic characteristics of the translational velocity, $\chi_{0}$, and angular velocity, $\sigma_{0}$ associated to $w_{0}$. We now recall that the equation $\mathcal{L}_{2}\left(w_{0}\right)+\beta \zeta_{0} w_{0}=\mathbf{0}$ is equivalent to the following set of equations

$$
\begin{gathered}
\left.\begin{array}{r}
-\Delta \boldsymbol{w}_{0}-\lambda_{\mathrm{c}} \boldsymbol{\tau}_{\mathrm{c}} \cdot \nabla \boldsymbol{w}_{0}+\lambda_{\mathrm{c}} L\left(\boldsymbol{w}_{0}\right)+\nabla \mathfrak{p}+\S \zeta \boldsymbol{w}_{0}=\mathbf{0} \\
\operatorname{div} \boldsymbol{w}_{0}=0
\end{array}\right\} \text { in } \Omega, \\
\boldsymbol{w}_{0}=\chi_{0}+\sigma_{0} \times \boldsymbol{x} \text { at } \partial \Omega, \\
\S \zeta M \chi_{0}+\int_{\partial \Omega} \mathbb{T}\left(\boldsymbol{w}_{0}, \mathfrak{p}\right) \cdot \boldsymbol{n}=\mathbf{0}, \quad \beta \zeta \mathcal{I} \sigma_{0}+\int_{\partial \Omega} \boldsymbol{x} \times \mathbb{T}\left(\boldsymbol{w}_{0}, \mathfrak{p}\right) \cdot \boldsymbol{n}=\mathbf{0},
\end{gathered}
$$

where

$$
L\left(\boldsymbol{w}_{0}\right):=\boldsymbol{v}_{\mathrm{c}} \cdot \nabla \boldsymbol{w}_{0}+\left(\boldsymbol{w}_{0}-\boldsymbol{\chi}_{0}\right) \cdot \nabla \boldsymbol{v}_{\mathrm{c}} .
$$

Thus, assuming the gravity directed along $\boldsymbol{e}_{1}$, an oscillatory motion of $G$ in the neighborhood of $\lambda=\lambda_{\mathrm{c}}$ will take place if and only if $\left(\chi_{0}\right)_{2} \boldsymbol{e}_{2}+\left(\chi_{0}\right)_{3} \boldsymbol{e}_{3} \neq \mathbf{0}$. In the remaining part of this section we shall furnish a characterization of the expression of $\chi_{0}$ and $\sigma_{0}$ that, in particular, will provide the desired property. 
Let us introduce the pairs $\left(\boldsymbol{h}^{(i)}, p^{(i)}\right)$ and $\left(\boldsymbol{H}^{(i)}, P^{(i)}\right)$ in $W^{2,2}(\Omega) \times D^{1,2}(\Omega)$, solutions to the following problems $(i=1,2,3)$ :

$$
\begin{gathered}
-\mathrm{i} \zeta_{0} \boldsymbol{h}^{(i)}+\lambda_{\mathrm{c}} \boldsymbol{\tau}_{\mathrm{c}} \cdot \nabla \boldsymbol{h}^{(i)}=\operatorname{div} \mathbb{T}\left(\boldsymbol{h}^{(i)}, p^{(i)}\right) \\
\operatorname{div} \boldsymbol{h}^{(i)}=0 \\
\boldsymbol{h}^{(i)}=\boldsymbol{e}_{i} \text { at } \partial \Omega,
\end{gathered} \text { in } \Omega
$$

and

$$
\begin{aligned}
& -\mathrm{i} \zeta_{0} \boldsymbol{H}^{(i)}+\lambda_{\mathrm{c}} \boldsymbol{\tau}_{\mathrm{c}} \cdot \nabla \boldsymbol{H}^{(i)}=\operatorname{div} \mathbb{T}\left(\boldsymbol{H}^{(i)}, P^{(i)}\right) \\
& \operatorname{div} \boldsymbol{H}^{(i)}=0 \\
& \boldsymbol{H}^{(i)}=\boldsymbol{e}_{i} \times \boldsymbol{x} \text { at } \partial \Omega .
\end{aligned} \text { in } \Omega
$$

Moreover, consider the matrices $\hat{\mathbb{K}}, \hat{\mathbb{A}}, \hat{\mathbb{P}}$, and $\hat{\mathbb{S}}$, defined by $(i, j=1,2,3)$

$$
\begin{gathered}
(\hat{\mathbb{K}})_{i j}=\int_{\Sigma}\left(\mathbb{T}\left(\boldsymbol{h}^{(i) *}, p^{(i) *}\right) \cdot \boldsymbol{n}\right)_{j} \\
(\hat{\mathbb{A}})_{i j}=\int_{\Sigma}\left(\boldsymbol{x} \times \mathbb{T}\left(\boldsymbol{H}^{(i) *}, P^{(i) *}\right) \cdot \boldsymbol{n}\right)_{j} \\
(\hat{\mathbb{P}})_{i j}=\int_{\Sigma}\left(\boldsymbol{x} \times \mathbb{T}\left(\boldsymbol{h}^{(i) *}, p^{(i) *}\right) \cdot \boldsymbol{n}\right)_{j} \\
(\hat{\mathbb{S}})_{i j}=\int_{\Sigma}\left(\mathbb{T}\left(\boldsymbol{H}^{(i) *}, P^{(i) *}\right) \cdot \boldsymbol{n}\right)_{j}
\end{gathered}
$$

where, we recall, * means complex conjugate. The existence of the above pairs in the specified function class is guaranteed by Lemma 7. Furthermore, again from this lemma, we know that the matrices $\mathbb{\mathbb { K }}+\beta \lambda \mathbb{1}$ and $\hat{\mathbb{A}}+\beta \mu \mathbb{1}$ are invertible, for all $\lambda, \mu \in \mathbb{R}$, as well as the block matrix

$$
\hat{\mathcal{A}}=\left(\begin{array}{cc}
\hat{\mathbb{K}}+\beta \lambda \mathbb{1} & \hat{\mathbb{P}} \\
\hat{\mathbb{S}} & \hat{\mathbb{A}}+\beta \mu \mathbb{1}
\end{array}\right) .
$$

If we dot-multiply both sides of $(111)_{1}$ by $\boldsymbol{h}^{(i) *}$, integrate by parts over $\Omega$, and employing $(111)_{2,3,4}$, we get

$$
B \zeta_{0}\left(\boldsymbol{w}_{0}, \boldsymbol{h}^{(i) *}\right)-\lambda_{\mathrm{c}}\left(\boldsymbol{\tau}_{\mathrm{c}} \cdot \nabla \boldsymbol{w}_{0}, \boldsymbol{h}^{(i) *}\right)+2\left[\mathbb{D}\left(\boldsymbol{w}_{0}\right), \mathbb{D}\left(\boldsymbol{h}^{(i) *}\right)\right]=-\S M \zeta_{0} \chi_{0} \cdot \boldsymbol{e}_{i}-\lambda_{\mathrm{c}}\left(L\left(\boldsymbol{w}_{0}\right), \boldsymbol{h}^{(i) *}\right) .
$$

Similarly, taking first the complex conjugate of $(113)_{1}$, then dot-multiplying it by $\boldsymbol{w}_{0}$, integrating by parts over $\Omega_{0}$, and using (115), we deduce

$$
B \zeta_{0}\left(\boldsymbol{w}_{0}, \boldsymbol{h}^{(i) *}\right)-\lambda_{\mathrm{c}}\left(\boldsymbol{\tau}_{\mathrm{c}} \cdot \nabla \boldsymbol{w}_{0}, \boldsymbol{h}^{(i) *}\right)+2\left[\mathbb{D}\left(\boldsymbol{w}_{0}\right), \mathbb{D}\left(\boldsymbol{h}^{(i) *}\right)\right]=\left[\hat{\mathbb{K}} \cdot \boldsymbol{\chi}_{0}+\hat{\mathbb{P}} \cdot \sigma_{0}\right]_{i} .
$$

From (116) and (117) we conclude (summation over repeated indeces)

$$
\widetilde{\mathbb{K}} \cdot \chi_{0}+\hat{\mathbb{P}} \cdot \sigma_{0}=-\lambda_{c}\left(L\left(\boldsymbol{w}_{0}\right), \boldsymbol{h}^{(i) *}\right) \boldsymbol{e}_{i}:=\mathcal{F},
$$

where $\widetilde{\mathbb{K}}:=\beta M \zeta_{0} \mathbb{1}+\widehat{\mathbb{K}}$. Likewise, we can show that

$$
\widetilde{\mathbb{A}} \cdot \sigma_{0}+\hat{\mathbb{S}} \cdot \chi_{0}=-\lambda_{\mathrm{c}}\left(L\left(\boldsymbol{w}_{0}\right), \boldsymbol{H}^{(i) *}\right) \boldsymbol{e}_{i}:=\boldsymbol{G},
$$

with $\widetilde{\mathbb{A}}:=ß \mathcal{I} \zeta_{0} \mathbb{1}+\hat{\mathbb{A}}$. From (118) and (119) we infer

$$
\chi_{0}=\mathbb{H} \cdot\left(\mathcal{F}+\widetilde{\mathbb{K}} \cdot \widetilde{\mathbb{A}}^{-1} \cdot \mathcal{G}\right), \quad \sigma_{0}=\mathbb{M} \cdot\left(\boldsymbol{G}+\widetilde{\mathbb{A}} \cdot \widetilde{\mathbb{K}}^{-1} \cdot \mathcal{F}\right),
$$

where

$$
\mathbb{H}:=\left(\widetilde{\mathbb{K}}-\hat{\mathbb{P}} \cdot \widetilde{\mathbb{A}}^{-1} \cdot \widehat{\mathbb{S}}\right)^{-1}, \quad \mathbb{M}:=\left(\widetilde{\mathbb{A}}-\widehat{\mathbb{S}} \cdot \widetilde{\mathbb{K}}^{-1} \cdot \hat{\mathbb{P}}\right)^{-1}
$$


Notice that both $\mathbb{H}$ and $\mathbb{M}$ exist, because $\hat{\mathcal{A}}, \widetilde{\mathbb{K}}$, and $\widetilde{\mathbb{A}}$ are invertible. From (120) we can then derive the following result.

Theorem 7. Suppose the assumptions of Theorem 6 hold. Then, an oscillatory motion of the center of mass $G$ of $\mathcal{S}$ in the neighborhood of $\lambda=\lambda_{\mathrm{c}}$ may occur if and only if

$$
\left[\mathbb{H} \cdot\left(\mathcal{F}+\widetilde{\mathbb{K}} \cdot \widetilde{\mathbb{A}}^{-1} \cdot \mathcal{G}\right)\right]_{2} \boldsymbol{e}_{2}+\left[\mathbb{H} \cdot\left(\mathcal{F}+\widetilde{\mathbb{K}} \cdot \widetilde{\mathbb{A}}^{-1} \cdot \boldsymbol{G}\right)\right]_{3} \boldsymbol{e}_{3} \neq \mathbf{0}
$$

Funding: This research received no external funding.

Institutional Review Board Statement: Not applicable.

Informed Consent Statement: Not applicable.

Data Availability Statement: Not applicable.

Conflicts of Interest: : The author declares that he has no conflict of interest.

\section{References}

1. Jenny, M.; Dušek, J.; Bouchet, G. Instabilities and transition of a sphere falling or ascending freely in a Newtonian fluid. J. Fluid Mech. 2004, 508, 201-239. [CrossRef]

2. Nakamura, I. Steady wake behind a sphere. Phys. Fluids 1976, 19, 5-8. [CrossRef]

3. Rao, M.A. Rheology of Fluid and Semisolid Foods Principles and Applications, 2nd ed.; Springer: Berlin/Heidelberg, Germany, 2014.

4. Schweizer, T. A quick guide to better viscosity measurements of highly viscous fluids. Appl. Rheol. 2004, 14, 197-201. [CrossRef]

5. Tachibana, M. The Transient Motion of a Falling Sphere in a Viscous Fluid and the Effects of Side Walls; Memoirs of Tile Faculty of Engineering, Fukui University: Fukui, Japan, 1976; Volume 24, pp. 157-169.

6. Taneda, S.; Kitasho, K. Experimental investigation of the wake behind a sphere at low Reynolds numbers. J. Phys. Soc. Jpn. 1956, 11, 1104-1108. [CrossRef]

7. Scoggins, J.R. Aerodynamics of spherical balloon wind sensors. J. Geophys. Res. 1964, 69, 591-598. [CrossRef]

8. Karamanev, D.; Chavarie, C.; Mayer, R. Dynamics of the free rise of a light solid sphere in liquid. AIChE J. 1996, 42, 1789-1792. [CrossRef]

9. Farwig, R.; Neustupa, J. On the spectrum of a Stokes-type operator arising from flow around a rotating body. Manuscripta Math. 2007, 122, 419-437. [CrossRef]

10. Babenko, K.I. On the Spectrum of a Linearized Problem on the Flow of a Viscous Incompressible Fluid around a Body (Russian); Dokl. Akad. Nauk; SSSR: Irvine, CA, USA, 1982; Volume 262, pp. 64-68.

11. Babenko, K.I. Periodic solutions of a problem of the flow of a viscous fluid around a body. Soviet Math. Dokl. 1982, 25, 211-216.

12. Haragus, M.; Iooss, G. Local Bifurcations, Center Manifolds, and Normal Forms in Infinite-Dimensional Dynamical Systems; Universitext; Springer: London, UK; EDP Sciences: Les Ulis, France, 2011.

13. Sattinger, D.H. Topics in Stability and Bifurcation Theory; Lecture Notes in Mathematics; Springer: Berlin, Germany; New York, NY, USA, 1973; Volume 309.

14. Zeidler, E. Nonlinear Functional Analysis and Applications; Application to Mathematical Physics; Springer: New York, NY, USA, 1988; Volume 4, Sections 72.7-72.9.

15. Galdi, G.P. Further properties of steady-state solutions to the Navier-Stokes problem past a three-dimensional obstacle. J. Math. Phys. 2007, 48, 065207. [CrossRef]

16. Galdi, G.P. A time-periodic bifurcation theorem and its applications to Navier-Stokes flow past an obstacle. In Mathematical Analysis of Viscous Incompressible Flow; Hishida, T., Kokyuroku, R.I.M.S., Eds.; Kyoto University: Kyoto, Japan, $2015 ;$ pp. 1-27.

17. Galdi, G.P. Steady-state Navier-Stokes problem past a rotating body: Geometric-functional properties and related questions. In Topics in Mathematical Fluid Mechanics; Springer: Berlin/Heidelberg, Germany, 2013; Volume 2073, pp. $109-197$.

18. Galdi, G.P. On bifurcating time-periodic flow of a Navier-Stokes liquid past a cylinder. Arch. Ration. Mech. Anal. 2016, 222, 285-315. [CrossRef]

19. Galdi, G.P. On the problem of steady bifurcation of a falling sphere in a Navier-Stokes liquid. J. Math. Phys. 2020, 61, 083101. [CrossRef]

20. Galdi, G.P. On the motion of a rigid body in a viscous liquid: A mathematical analysis with applications. In Handbook of Mathematical Fluid Dynamics; Elsevier: Amsterdam, The Netherlands, 2002; Volume I, 653-791.

21. Silvestre, A.L. On the self-propelled motion of a rigid body in a viscous liquid and on the attainability of steady symmetric self-propelled motions. J. Math. Fluid Mech. 2002, 4, 285-326. [CrossRef]

22. Galdi, G.P. An Introduction to the Mathematical Theory of the Navier-Stokes Equations. Steady-State Problems, 2nd ed.; Springer Monographs in Mathematics; Springer: New York, NY, USA, 2011.

23. Gohberg, I.; Goldberg, S.; Kaashoek, M.A. Classes of Linear Operators: I. Operator Theory, Advances and Applications; Birkhäuser: Basel, Switzerland, 1990; Volume 49. 
24. Heywood, J.G. The Navier-Stokes equations: On the existence, regularity and decay of solutions. Indiana Univ. Math. J. 1980, 29, 639-681. [CrossRef]

25. Galdi, G.P. On the self-propulsion of a rigid body in a viscous liquid by time-periodic boundary data. J. Math. Fluid Mech. 2020, 22, 1-34. [CrossRef]

26. Galdi, G.P.; Kyed, M. Time-periodic solutions to the Navier-Stokes equations. In Handbook of Mathematical Analysis in Mechanics of Viscous Fluids; Springer: Cham, Switzerland, 2018; pp. 509-578.

27. Solonnikov, V.A. Estimates of the solutions of the nonstationary Navier-Stokes system. Zap. Naucn. Sem. Leningrad. Otdel. Mat. Inst. Steklov. (LOMI) 1973, 38, 153-201. 